\title{
An embedded approach for immiscible multi-fluid problems
}

\author{
P. B. Ryzhakov and A. Jarauta
}

\begin{abstract}
An embedded formulation for the simulation of immiscible multi-fluid problems is proposed. The method is particularly designed for handling gas-liquid systems. Gas and liquid are modeled using the Eulerian and the Lagrangian formulation, respectively. The Lagrangian domain (liquid) moves on top of the fixed Eulerian mesh. The location of the material interface is exactly defined by the position of the boundary mesh of the Lagrangian domain. The individual fluid problems are solved in a partitioned fashion and are coupled using a Dirichlet-Neumann algorithm. Representation of the pressure discontinuity across the interface does not require any additional techniques being an intrinsic feature of the method. The proposed formulation is validated and its potential applications are shown.
\end{abstract}

\section{Introduction}

Multi-fluid systems are encountered in a large number of natural and industrial processes. This work focuses on the immiscible incompressible liquid-gas systems, frequently found in civil and mechanical engineering. By far, the most common two-fluid system in these applications is water-air. Such systems are representative for e.g. free-surface flow problems whenever the effects of air are not negligible (e.g. water sloshing) and pipe flows in the presence of water (e.g. water droplets exposed to airflow).

In spite of the fact that multi-fluid flows are frequently encountered in nature and engineering practice, they still pose a major research challenge from both theoretical and computational points of view. Multi-fluid problems require not only modeling of the physical constituents (each of the fluids), but also the interaction between them. The main challenges of a multi-fluid simulation consist in a) detecting the interface between fluids b) accounting for the discontinuities in the material properties (density, viscosity) across this interface. Modeling of these challenging aspects is closely related to the kinematic frameworks chosen for each fluid.

State-of-the-art Several strategies for immiscible multi-fluid simulation have been developed in the past. One such strategy relies on using Eulerian approach. In such case both fluids are solved using a single fixed mesh. The fluid interface crosses the elements of the fixed mesh at arbitrary positions and must be determined by special interface capturing techniques. These techniques provide an additional equation describing the evolution of the interface. One typical choice 
is the level-set approach, a method based on a convection of the smooth distance function [1], [2]. Zero of the distance function represents the interface position. Similarly, Volume of Fluid (VOF) methods introduce an convection equation for the discontinuous volume fraction function [3]. Combination of an Eulerian formulation with a moving Lagrangian interface can be found in [4]. In all these approaches the conservation equations of both fluids ares solved simultaneously in a single equations system, while the interface equation is solved in a separate step and may violate the conservation. Thus, the majority of the interface capturing techniques exhibit deficiencies in conserving the mass. Several recent studies have introduced novel techniques for alleviating this deficiency [5], [6], [7], [8], [9].

Fixed mesh approaches also require special techniques for representing the discontinuity of the material properties across the interface. These include pressure and/or velocity enrichment or discontinuous interpolations for the interface elements [10], [9]. The enhanced integration techniques are necessary to adequately represent the contribution of the interface elements to the governing system of equations.

Another strategy for immiscible multi-fluid simulation relies on solving the heterogeneous system monolithically in a Lagrangian fashion [11], [12]. In such case the interface coincides with the position of the nodes of the moving computational mesh and its evolution is defined by the solution of the flow problem.

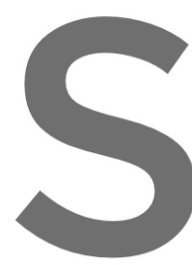

Thus, no addit

position.

(often encou

to represent th

be duplicated

Similarl
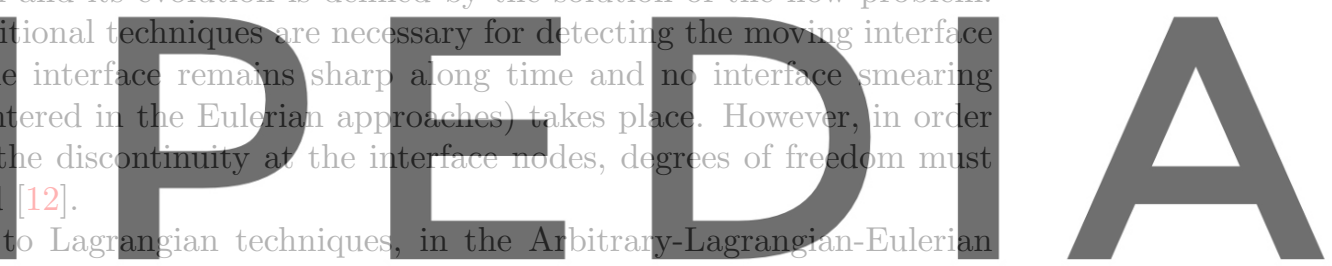

(ALE) approaches the interface position is identified by the computational mesh

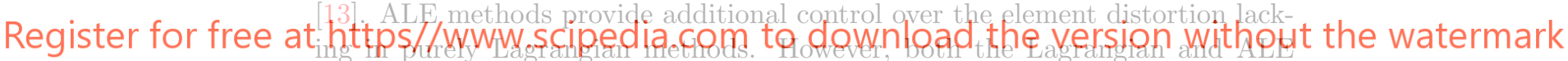

methods allow for only moderate mesh deformations without falling into element degradation. The re-meshing may lead to difficulties in preserving the interface. If no special care is taken, the swapping of interface elements' edges may lead to interface "break-up".

Yet there exists another possibility, the embedded approach ${ }^{1}$, often used in the field of fluid-structure interaction (FSI) modeling (see e.g. [14]), but merely addressed in the multi-fluid context. It relies on combining an Eulerian and a Lagrangian description for the individual fluids of a multi-fluid problem and solving them in a partitioned manner. A fluid described by a Lagrangian formulation can be naturally embedded into an Eulerian mesh similarly to Lagrangian structures embedded into Eulerian fluid in the FSI methods ${ }^{2}$. According to the embedded strategy applied to the gas-liquid systems the Lagrangian sub-domain (liquid) is moving on top of the fixed Eulerian mesh forcing the gas to deform accordingly and receiving the feedback in terms of stresses at the interface boundary.

For problems involving motion of liquid in gas, it is advantageous to use the

\footnotetext{
${ }^{1}$ These are also known in the literature under the names such as "immersed boundary" or "fictitious domain" methods.

${ }^{2}$ This holds for immiscible fluids. In case of miscible fluids the embedded approach is not straightforward, as the phases in such cases cannot be clearly separated and are not conserved
} 


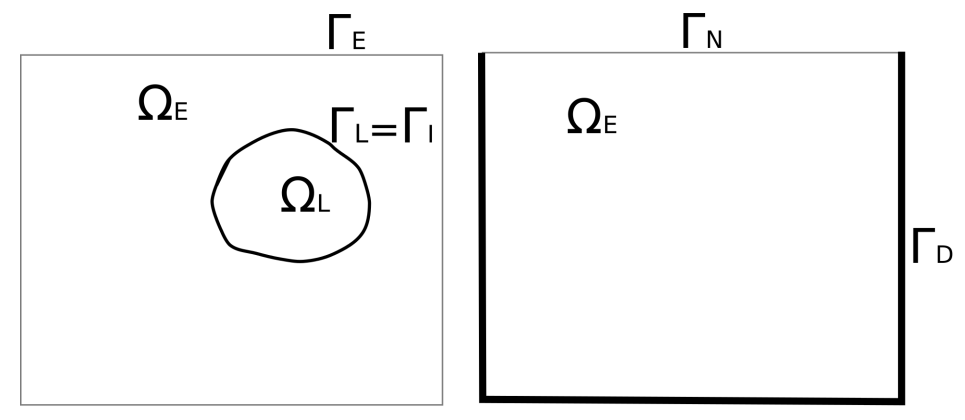

(a) Schematic representation of the (b) Dirichlet and Neumann parts of exembedded two-fluid system ternal boundary $\Gamma_{E}$

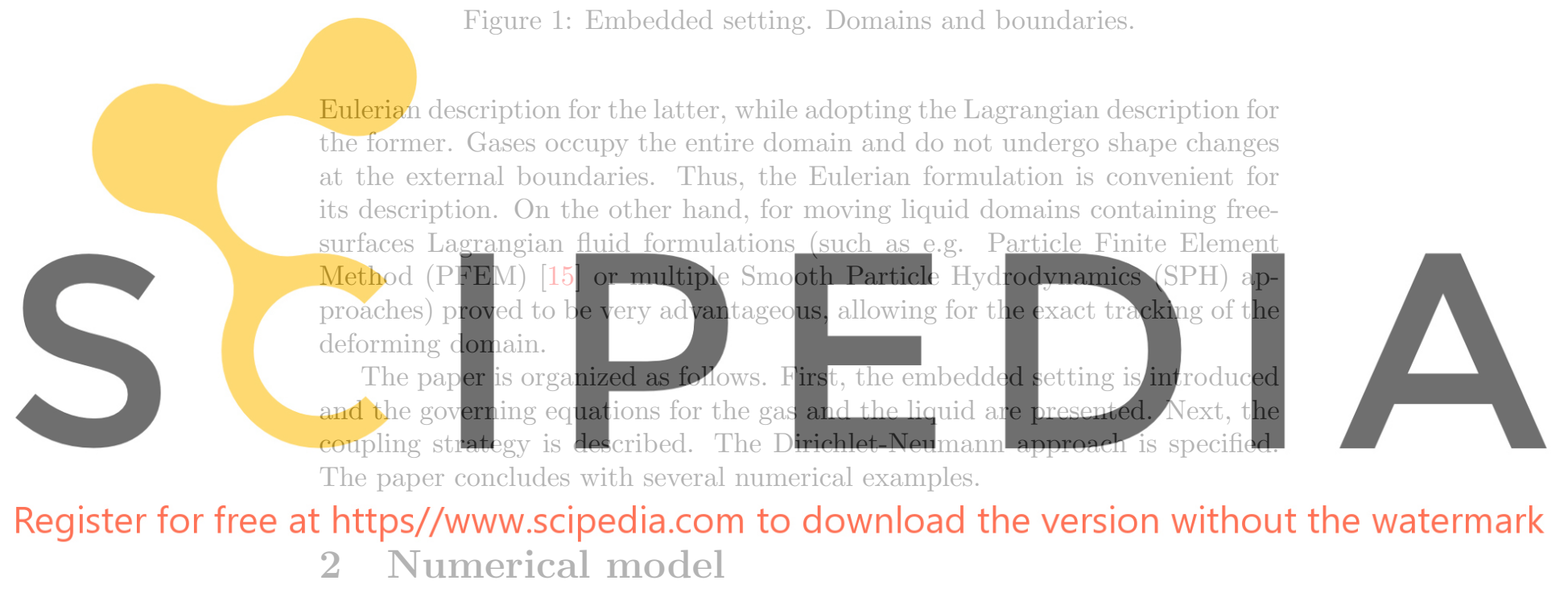

\subsection{Governing equations}

Let us consider Lagrangian domain $\Omega_{L}$ (representing liquid) embedded into the Eulerian domain $\Omega_{E}$ with an external boundary $\Gamma_{E}$ (see Fig. 1(a)). In the embedded setting the interface $\Gamma_{I}$ between the two fluids is defined by the position of the boundary of the Lagrangian domain $\Gamma_{L}$. The interface $\Gamma_{I}$ splits the Eulerian domain into two parts: the real one $\Omega_{E}^{r}$ (representing the gas) and the fictitious one $\Omega_{E}^{f}$ that does not have physical meaning. Note that at the continuous level the fictitious Eulerian domain exactly coincides with the Lagrangian domain and $\Gamma_{I}=\Gamma_{L}$. Next we present the governing equations for the gas and the liquid domains.

Let us assume that both substances are incompressible Newtonian fluids. The governing system in either domain is therefore the Navier-Stokes equations equipped with the incompressibility condition: 


$$
\begin{array}{ll}
\rho \frac{D \mathbf{v}}{D t}-\mu \nabla \cdot\left(\nabla \mathbf{v}+\nabla^{T}(\mathbf{v})\right)+\nabla p=\rho \mathbf{g} & \text { in } \Omega_{E}^{r} \text { and } \Omega_{L} \\
\nabla \cdot \mathbf{v}=0 & \text { in } \Omega_{E}^{r} \text { and } \Omega_{L}
\end{array}
$$

where $\frac{D \mathbf{v}}{D t}$ is the material time derivative of the velocity, $p$ is the pressure, $t$ is the time, $\mathbf{g}$ is the body force, $\rho$ is the fluid density and $\mu$ is the fluid dynamic viscosity. The physical properties are defined as $\rho=\rho_{E}$ in $\Omega_{E}$ and $\rho=\rho_{L}$ in $\Omega_{L}$.

Boundary and interface conditions In order to ensure the well-posedness of the Navier-Stokes problem defined by Eqs. (1), (2) suitable boundary conditions must be specified. On the external boundary $\Gamma_{E}=\Gamma_{D} \cup \Gamma_{N}$, such that $\Gamma_{D} \cap \Gamma_{N}=\emptyset$, the following conditions are prescribed:
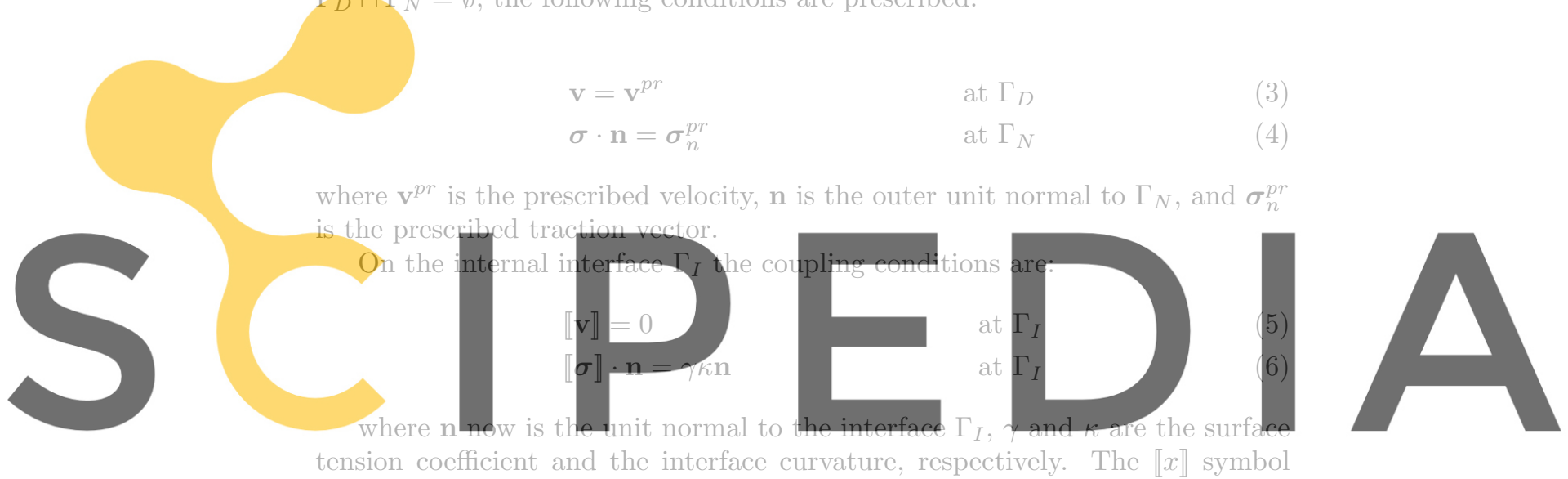

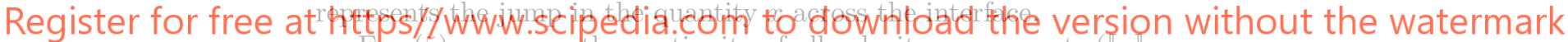

Eq. (5) expresses the continuity of all velocity components $\left(\llbracket \mathbf{v} \rrbracket=\mathbf{v}_{E}-\mathbf{v}_{L}\right.$,

where indices $E$ and $L$ distinguish the quantities corresponding to the Eulerian fluid (gas) and the Lagrangian fluid (iiquid), respectively). The equality of the normal components of velocity ensures no mass flow across the interface. The tangential components' equality is similar to a no-slip condition and is necessary when fluids with non-zero viscosity are considered. Eq. (6) expresses that the difference in the normal stress across the interface is balanced by the surface tension force.

Projecting Eq. (6) onto the normal and tangential directions leads to the following scalar interface conditions:

$$
\begin{array}{ll}
\mathbf{n} \cdot \llbracket \boldsymbol{\sigma} \rrbracket \cdot \mathbf{n}=\gamma \kappa & \text { at } \Gamma_{I} \\
\mathbf{t} \cdot \llbracket \boldsymbol{\sigma} \rrbracket \cdot \mathbf{n}=0 & \text { at } \Gamma_{I}
\end{array}
$$

Noting that the jump in the stress across the interface is equal to the difference between the stresses of the two fluids $\llbracket \boldsymbol{\sigma} \rrbracket=\sigma_{E}-\boldsymbol{\sigma}_{L}$ and splitting the stress tensor into volumetric and deviatoric part as $\boldsymbol{\sigma}=-p \boldsymbol{I}+2 \mu \frac{\nabla \mathbf{v}+\nabla^{T} \mathbf{v}}{2}$ results in:

$$
\begin{aligned}
& \left(p_{E}-p_{L}\right)+\mu_{E} \mathbf{n} \cdot\left[\nabla \mathbf{v}+\nabla^{T} \mathbf{v}\right]_{E} \cdot \mathbf{n}-\mu_{L} \cdot \mathbf{n} \cdot\left[\nabla \mathbf{v}+\nabla^{T} \mathbf{v}\right]_{L} \cdot \mathbf{n}=\gamma \kappa \\
& \mu_{E} \mathbf{t} \cdot\left[\nabla \mathbf{v}+\nabla^{T} \mathbf{v}\right]_{E} \cdot \mathbf{n}-\mu_{L} \cdot \mathbf{t} \cdot\left[\nabla \mathbf{v}+\nabla^{T} \mathbf{v}\right]_{L} \cdot \mathbf{n}=0
\end{aligned}
$$




\subsection{Finite Element formulation for the gas}

The equal order linear velocity/pressure interpolations over 3-noded triangles (2D) or 4-noded tetrahedra (3D) are used here for the space discretization of the governing equations Eqs. (1), (2). We assume Backward Euler time discretization scheme exclusively for the sake of simplicity. All the arguments presented in the paper are valid for any implicit time integration scheme. Being standard, the space and time discretization are not discussed here (see e.g. [16], [17]).

Given $\overline{\mathbf{v}}_{n}$ and $\bar{p}_{n}$ at $t_{n}$, the time discrete problem consists in finding $\overline{\mathbf{v}}_{n+1}$ and $\bar{p}_{n+1}$ at $t_{n+1}$ as the solution of

$$
\begin{aligned}
& \mathbf{M} \frac{\overline{\mathbf{v}}_{n+1}-\overline{\mathbf{v}}_{n}}{\Delta t}+\left[\overline{\mathbf{K}}\left(\overline{\mathbf{v}}_{n+1}\right)+\mu \mathbf{L}\right] \overline{\mathbf{v}}_{n+1}+\mathbf{G} \bar{p}_{n+1}=\overline{\mathbf{F}} \\
& \mathbf{D} \overline{\mathbf{v}}_{n+1}=0
\end{aligned}
$$

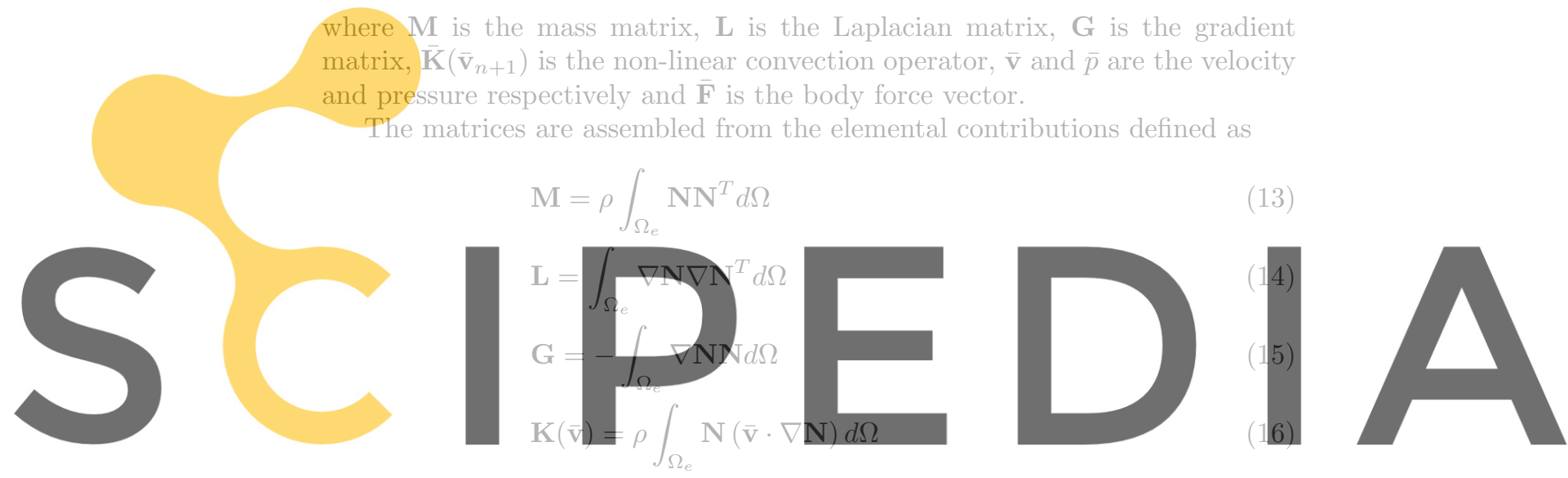

Register for free at https//www.

$$
\mathrm{D}=-\mathrm{G}^{T}
$$

$\mathbf{N}$ stands for the vector of standard linear FE shape functions, $\Omega_{e}$ is the element integration domain.

The conditions at the external boundary are: $\overline{\mathbf{v}}=0$ at $\Gamma_{D}$ and $\boldsymbol{\sigma} \cdot \mathbf{n}=0$ at $\Gamma_{N}$ ( see Fig.1(b)).

It is well-known that the governing system defined by Eqs. (11),(12) must be stabilized (convection stabilization and inf-sup stabilization of the incompressibility constraint for equal order velocity-pressure elements). Discussing details of the stabilization lies outside of scope of this work and the ideas presented here can be applied in conjunction with practically any stabilization technique. In the present work Algebraic Sub-Grid Scales (ASGS) stabilization [18] is implemented. The stabilized equations are:

$$
\begin{aligned}
& \mathbf{M} \frac{\overline{\mathbf{v}}_{n+1}-\overline{\mathbf{v}}_{n}}{\Delta t}+\left[\overline{\mathbf{K}}\left(\overline{\mathbf{v}}_{n+1}\right)+\mu \mathbf{L}+\mathbf{S}_{K}\right] \overline{\mathbf{v}}_{n+1}+\left[\mathbf{G}+\mathbf{S}_{G}\right] \bar{p}_{n+1}=\overline{\mathbf{F}} \\
& \mathbf{D} \overline{\mathbf{v}}_{n+1}+\mathbf{S}_{D} \bar{p}_{n+1}=0
\end{aligned}
$$


The stabilization matrices are defined as (see [18] for details):

$$
\begin{aligned}
& \mathbf{S}_{K}=\int_{\Omega_{e}}\left(\overline{\mathbf{v}}_{n+1} \cdot \nabla \mathbf{N}\right) \tau\left(\frac{\rho}{\Delta t} \mathbf{N}+\rho \overline{\mathbf{v}}_{n+1} \cdot \nabla \mathbf{N}\right) \\
& \mathbf{S}_{G}=\int_{\Omega_{e}}\left(\overline{\mathbf{v}}_{n+1} \cdot \nabla \mathbf{N}\right) \tau \nabla \mathbf{N} d \Omega \\
& \mathbf{S}_{D}=\int_{\Omega_{e}}(\nabla \mathbf{N}) \tau\left(\frac{\rho}{\Delta t} \mathbf{N}+\rho \overline{\mathbf{v}}_{n+1} \cdot \nabla \mathbf{N}\right) d \Omega
\end{aligned}
$$

where $\tau$ is an algorithmic stabilization coefficient defined as $\tau=\left(\frac{2\|\overline{\mathbf{v}}\|}{h}+\frac{4 \nu}{h^{2}}\right)^{-1}$, where $h$ is the element size.

For reducing the computational cost associated to the solution of the governing system, the fractional splitting is applied. Fractional step or pressure projection approach (see [19], [20] or [21] ) uncouples the velocity and the pressure. Instead of one large and poorly conditioned system of equations two smaller and better conditioned systems are solved. Fractional step approach is applied here at a purely algebraic level (according to [22]), splitting the momentum equation Eqs. (19) into two parts by introducing the intermediate velocity $\tilde{\mathbf{v}}$. Thus, the original monolithic system Eqs. (19), (20) is replaced by
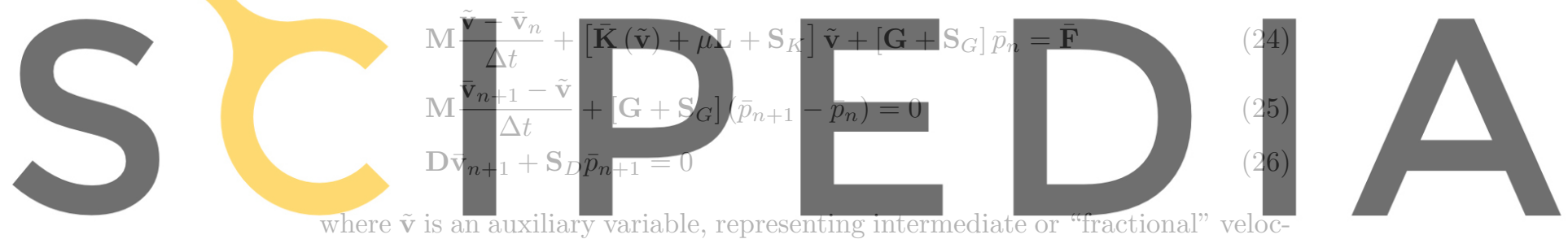

ity. Eq. (24) is known as "fractional momentum" and Eq. (25) as "end-of-step Register for free atnhttosh/wwawasioipedia.com to download the version without the watermark

The pressure Poisson's equation is obtained by applying the incompressibility condition Eq. (26) to the end-of-step momentum equation, leading to

$$
\mathbf{D} \tilde{\mathbf{v}}=\left[\Delta t \mathbf{D M}^{-1} \mathbf{G}-\mathbf{S}_{D}\right]\left(\bar{p}_{n+1}-\bar{p}_{n}\right)
$$

The term $\mathbf{D} \mathbf{M}^{-1} \mathbf{G}$ can approximated as $\mathbf{D} \mathbf{M}^{-1} \mathbf{G} \approx \mathbf{L}$ as the former one requires performing global matrix-vector multiplications and is, thus, computationally more expensive. Therefore, Eq. (27) obtains the following form:

$$
\mathbf{D} \tilde{\mathbf{v}}=\left[\Delta t \mathbf{L}-\mathbf{S}_{D}\right]\left(\bar{p}_{n+1}-\bar{p}_{n}\right)
$$

In order to overcome the singularity of the Laplacian matrix $\mathbf{L}$, an essential boundary condition for the pressure $p=0$ at $\Gamma_{N}$ is specified. Eqs. (24), (25) and (28) define the set of discrete governing equations in the Eulerian domain. The fractional momentum equation is non-linear due to the dependence of the convective term (and the corresponding stabilization terms) upon the unknown velocity. The fixed point iteration method is applied for their solution. Once Eq. (24) is solved for the fractional velocity $\tilde{\mathbf{v}}$, pressure $\bar{p}_{n+1}$ is obtained by solving Eq. (28) and finally the end-of-step velocity $\overline{\mathbf{v}}_{n+1}$ is obtained using Eq. (25). 


\subsection{Finite Element formulation for the liquid}

A powerful class of Lagrangian methods capable of liquid flow modeling is the Particle Finite Element Method [15], [23]. It allows to naturally track the motion of boundaries and interfaces. In the present work we adopt the basic features of the PFEM in the model for the liquid.

The PFEM describes the fluid in an updated Lagrangian framework. The mesh nodes are treated as particles that can freely move and even separate from the main fluid domain. The key idea of the PFEM is that the variables are stored at the nodes instead of the Gauss points. This results in a hybrid between a standard FE and a mesh-free method. A finite element mesh is created at every time step of the dynamic problem and the solution is then stored at the nodes. The nodes move according to their velocity to the new positions and then the finite element mesh is re-generated using Delaunay triangulation [24]. For further details on the PFEM approach the reader may refer to [15].

The discrete version of the governing equations (Eq. (1) and (2)) in the Lagrangian framework is given by:
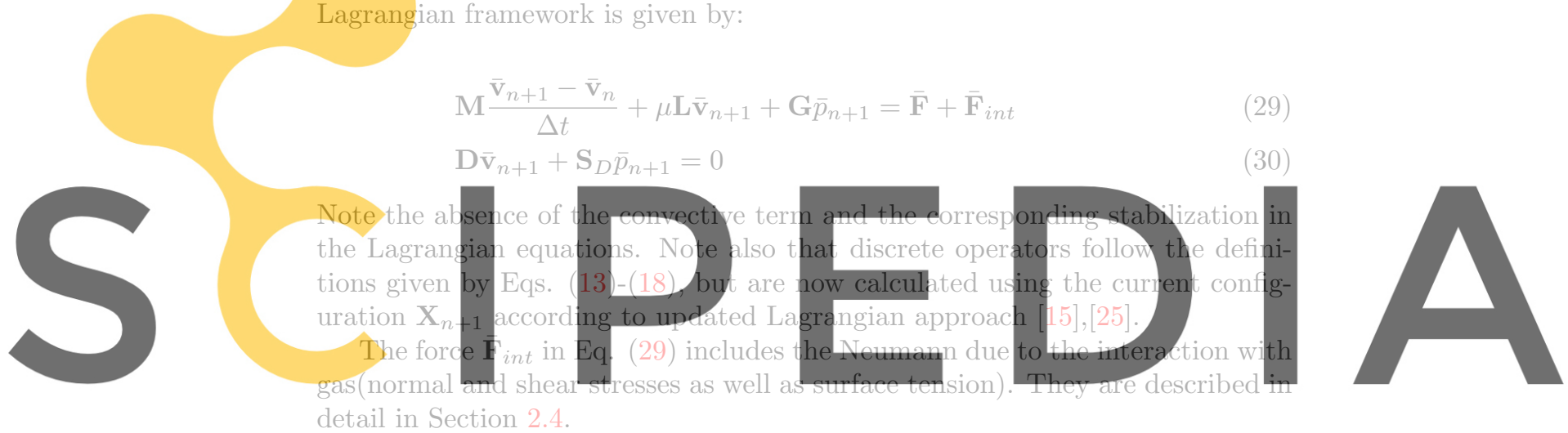

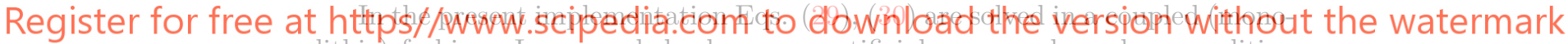

lithic) fashion. In a coupled scheme no artificial pressure boundary condition

at $\Gamma_{L}$ needs to be prescribed ${ }^{3}$. Thus, violating the incompressibility condition

at the boundary is avoided. In case of the fractional step method, the position of the boundary nodes corresponding to the fractional (non-divergence free) velocity alter the curvature of the boundary and consequently, the surface tension force. We show in Section(3.1) that this may lead to the spurious oscillations at the boundary. Note, however, that solving the governing system in a coupled fashion is not the only remedy. Any technique not requiring inconsistent pressure boundary condition can be applied. Interesting alternatives include the use of non-singular approximations of the Laplacian matrix in the pressure Poisson's equation or the schemes based on consistent pressure boundary condition (e.g. incremental pressure-correction scheme in rotational form, see [21]).

Eqs. (29), (30) are non-linear since in the updated Lagrangian setting the discrete operators (Eqs. (13)-(18)) correspond to the unknown current configuration $\left(\mathbf{X}_{n+1}\right)$. Therefore, the governing equations' system must be solved iteratively, updating the operators at every iteration $i$.

\footnotetext{
${ }^{3}$ In the fractional step technique described in this paper, fixing pressure is necessary due to the presence of the singular Laplacian matrix. At the nodes where the pressure is fixed, the end-of-step momentum equation does not provide the velocity correction since the difference between the current and historical pressure is zero (see Eq. 25)). Therefore, the fractional velocity persists at the boundary
} 
Let us define the residuals of the momentum and the continuity equations:

$$
\begin{aligned}
& \overline{\mathbf{r}}_{m}=\overline{\mathbf{F}}+\overline{\mathbf{F}}_{i n t}-\left[\mathbf{M} \frac{\overline{\mathbf{v}}_{n+1}^{i}-\overline{\mathbf{v}}_{n}}{\Delta t}+\mu \mathbf{L} \overline{\mathbf{v}}_{n+1}^{i}+\mathbf{G} \bar{p}_{n+1}^{i}\right] \\
& \overline{\mathbf{r}}_{c}=-\mathbf{D} \overline{\mathbf{v}}_{n+1}^{i}-\mathbf{S}_{D} \bar{p}_{n+1}^{i}
\end{aligned}
$$

The governing system in the residual form can thus be written as:

$$
\left(\begin{array}{cc}
\frac{\mathbf{M}}{\Delta t}+\mu \mathbf{L} & \mathbf{G} \\
\mathbf{D} & \mathbf{S}_{D}
\end{array}\right)\left(\begin{array}{l}
d \overline{\mathbf{v}} \\
d \bar{p}
\end{array}\right)=\left(\begin{array}{c}
\overline{\mathbf{r}}_{m} \\
\overline{\mathbf{r}}_{c}
\end{array}\right)
$$

Once the system (Eq. (33)) is solved, the velocity and the pressure are updated as $\overline{\mathbf{v}}_{n+1}^{i+1}=\overline{\mathbf{v}}_{n+1}^{i}+d \overline{\mathbf{v}}$ and $\bar{p}_{n+1}^{i+1}=\bar{p}_{n+1}^{i}+d \bar{p}$. The position of the nodes in $\Omega_{L}$ is updated as $\mathbf{X}_{n+1}^{i+1}=\mathbf{X}_{n}+\Delta t \cdot \overline{\mathbf{v}}_{n+1}^{i+1}$

The integration domains necessary for the computation of the discrete operators (Eqs. (13)-(18)) are be updated according to this new configuration $\mathbf{X}_{n+1}^{i+1}$. Once the convergence of the iterative loop was achieved and the end-of-step velocity $\left(\overline{\mathbf{v}}_{n+1}\right)$ and pressure $\left(\bar{p}_{n+1}\right)$ were obtained, the definitive mesh position is computed as
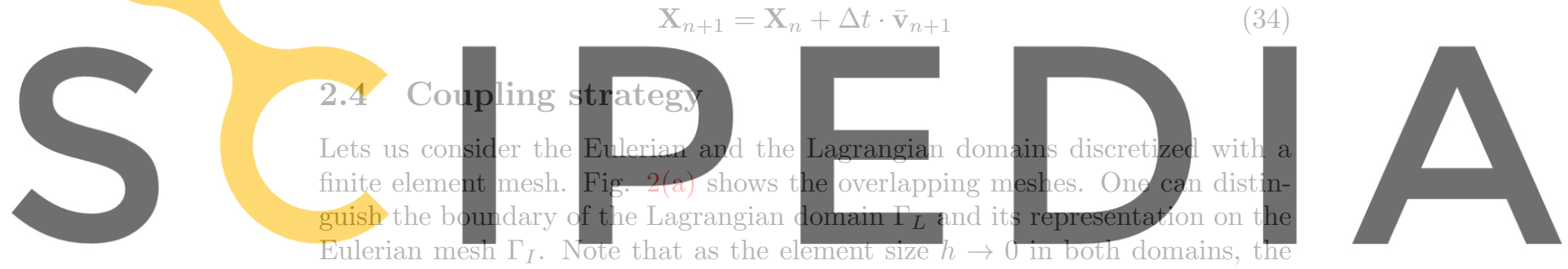

two boundaries tend to coincide: $\Gamma_{I} \rightarrow \Gamma_{I}$. The embedded interface $\Gamma_{I}$ (see

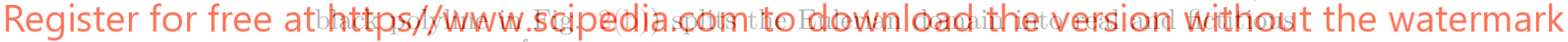
parts $\Omega_{E}^{r}$ and $\Omega_{F}^{f}$, as already mentioned. We shall denominate the nodes and the elements contained in $\Omega_{E}^{r}$ and $\Omega_{E}^{f}$ "real" and "fictitious, respectively (see Fig. 2(b)). We also distinguish the "interface elements", i. e. the elements cut by $\Gamma_{I}$ and thus containing both the real and the fictitious nodes. This is shown in Fig. 2(c), where the interface elements are shown in gray, fictitious and real nodes are indicated by black and gray dots, respectively.

For the coupling of the sub-problems we propose a Dirichlet-Neumann coupling technique. The action of the liquid (Lagrangian fluid) onto gas (Eulerian fluid) is represented via the Dirichlet boundary condition at $\Gamma_{I}$ ensuring the interface condition defined by Eq. (5). The feedback of the gas is represented by the interface Neumann b.c. applied at the immersed liquid boundary $\Gamma_{L}$. This b.c. represents the interface condition defined by Eqs. (9) and (10)).

Dirichlet boundary condition The interface $\Gamma_{I}$ intersects the Eulerian mesh at arbitrary positions, not necessarily at the nodes (see Fig. 2). Therefore in order to apply the interface Dirichlet boundary condition $\left(\mathbf{v}_{E}=\mathbf{v}_{L}\right.$ at $\left.\Gamma_{I}\right)$ two options exist. First one consists in sub-dividing the Eulerian elements, so that the intersections between the Lagrangian boundary $\Gamma_{L}$ and Eulerian mesh coincide with the newly introduced nodes. This choice permits applying the boundary condition in a "strong way" by prescribing the velocity of 

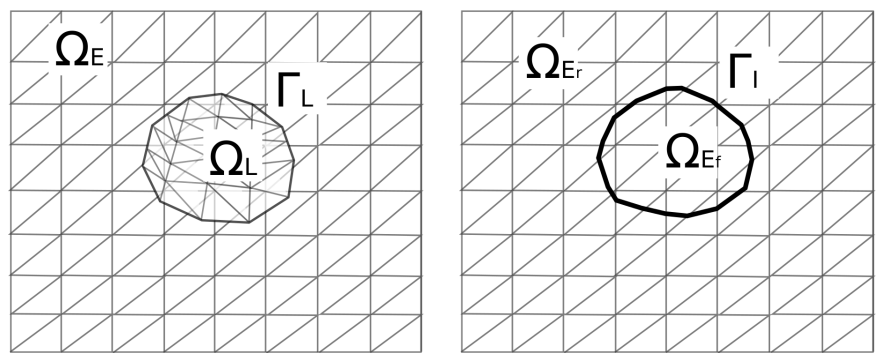

(a) Superimposed discretized do- (b) Interface on the Eulerian mesh mains

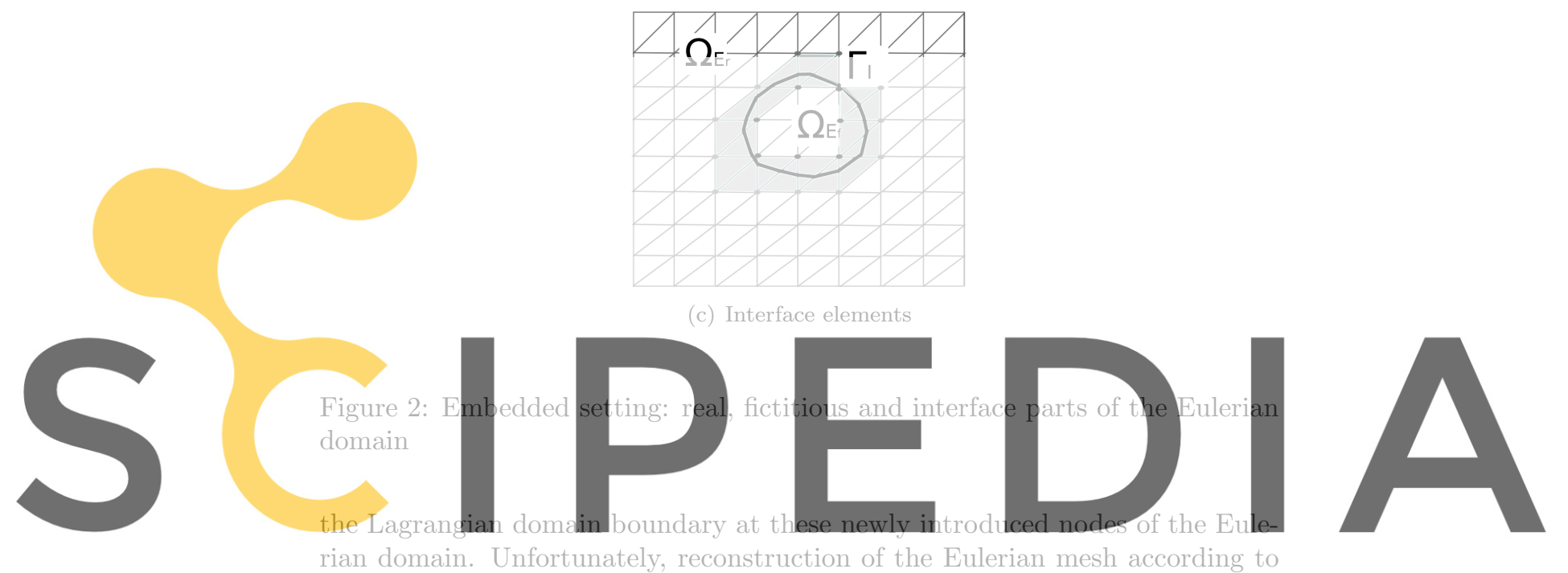

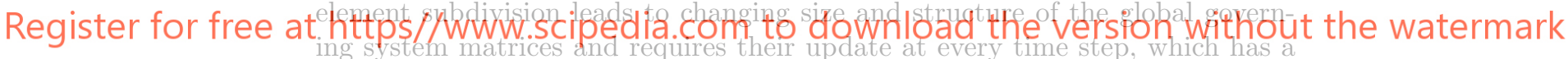
negative effect upon the computational efficiency.

The second option relies on applying the interface Dirichlet boundary condition in a "weak" sense, i.e. minimizing the difference between the velocity of the Lagrangian and Eulerian domain at the interface. in the integral sense along the interface $\Gamma_{I}$. The interface Dirichlet boundary condition can thus be applied at the existing fictitious nodes of the interface elements ${ }^{4}$ (see black dots in the interface elements in Fig. 2(c)). This way, only the existing degrees of freedom are used and thus the global matrix structure corresponding the Eulerian domain remains unaltered [26].

Given the velocity $\mathbf{v}_{L}$ of the liquid at its boundary, the difference to be minimized is given by (see e.g. [27] or [28] for details)

$$
\int_{\Gamma_{I}} \omega\left(\mathbf{v}_{n+1}-\mathbf{v}_{L}\right) d \Gamma_{I}=0
$$

where $\mathbf{v}_{n+1}$ and $\mathbf{v}_{L}$ are the velocities of the Eulerian and Lagrangian domains at the interface, respectively and $\omega=\mathbf{N}^{T} q$ is the velocity test function.

\footnotetext{
${ }^{4}$ This can be understood as: apply a velocity at fictitious nodes of the Eulerian interface elements, such that the difference between the resulting velocity of the Eulerian fluid and the liquid surface is zero in the least square sense.
} 
After velocity discretization $(\mathbf{v}=\mathbf{N} \overline{\mathbf{v}})$ Eq. (35) leads to:

$$
\mathbf{M}_{\Gamma_{I}} \overline{\mathbf{v}}_{n+1}=\mathbf{f}_{\Gamma_{I}}
$$

where $\overline{\mathbf{v}}_{n+1}$ is the velocity of the nodes of the interface elements, $\mathbf{M}_{\Gamma_{I}}=\int_{\Gamma_{I}} \mathbf{N}^{T} \mathbf{N} d \Gamma_{I}$ and $\mathbf{f}_{\Gamma_{I}}=\int_{\Gamma_{I}} \mathbf{N}_{f}^{T} \mathbf{v}_{L} d \Gamma_{I}$, where $N_{f}$ are the shape functions corresponding to the fictitious nodes. Considering that the interface elements contain both "real" and "fictitious" nodes (with a certain abuse of notation $\overline{\mathbf{v}}_{n+1}=\overline{\mathbf{v}}_{n+1, r}+\overline{\mathbf{v}}_{n+1, f}$, assuming that the entries in the vectors of the "real" nodal velocities corresponding to the "fictitious" nodes are zero and vice versa ${ }^{5}$ ), one can rewrite Eq. (36) as

$$
\mathbf{M}_{\Gamma_{I}} \overline{\mathbf{v}}_{n+1, r}+\mathbf{M}_{\Gamma_{I}} \overline{\mathbf{v}}_{n+1, f}=\mathbf{f}_{\Gamma_{I}}
$$

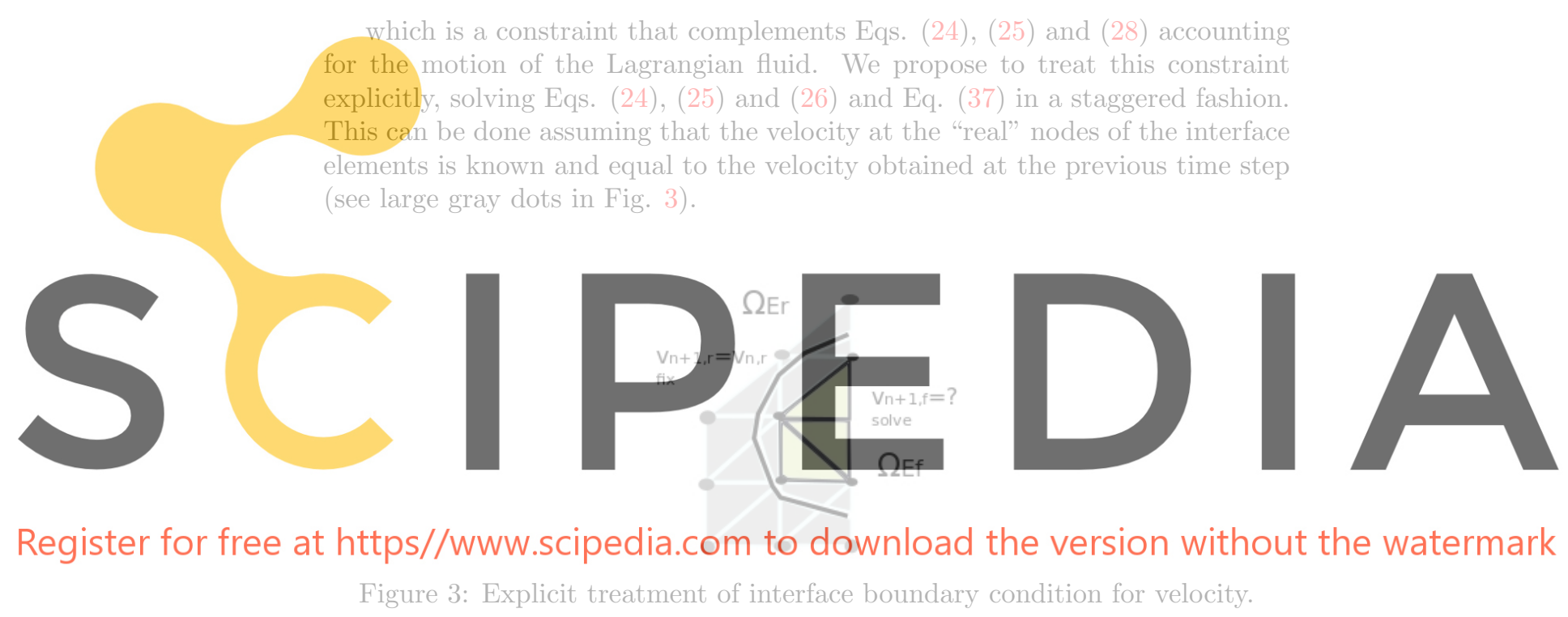

This can be expressed as

$$
\begin{array}{r}
\mathbf{M}_{\Gamma_{I}} \overline{\mathbf{v}}_{n+1, f}=\mathbf{f}_{\Gamma_{I}}-\mathbf{M}_{\Gamma_{I}} \overline{\mathbf{v}}_{n+1, r} \\
\overline{\mathbf{v}}_{n+1, r} \approx \overline{\mathbf{v}}_{n, r}
\end{array}
$$

where $n$ corresponds to the time step index. Solving Eq. (38) the velocity at the fictitious nodes is obtained (see black dots in Fig. 3), that is consequently applied as the interface Dirichlet boundary condition at the next time step, representing the action of the Lagrangian fluid onto the Eulerian one. Note that the fully fictitious fluid elements are excluded from the system.

Neumann boundary condition Interface Neumann boundary condition represents the normal and shear stress components exerted by the gas upon the liquid as well as the surface tension. It corresponds the interface condition defined by Eqs. (7) and (8). It is accounted for by the term $\overline{\mathbf{F}}_{\text {int }}$ (Eq. (40)) in

\footnotetext{
${ }^{5}$ for example, let us consider a triangular interface element, whose nodes 1 and 3 are "real" and 2 is "fictitious". Then $\overline{\mathbf{v}}_{r}=\left[\overline{\mathbf{v}}_{1}, 0, \overline{\mathbf{v}}_{3}\right]^{T}$ and $\overline{\mathbf{v}}_{f}=\left[0, \overline{\mathbf{v}}_{2}, 0\right]^{T}$
} 
the momentum Eq. (29) of the liquid. This term is computed as a sum of the following contributions:

$$
\overline{\mathbf{F}}_{i n t}=\overline{\mathbf{F}}_{N}+\overline{\mathbf{F}}_{s t}+\overline{\mathbf{F}}_{s h} \quad \text { on } \Gamma_{L}
$$

where

$$
\begin{array}{ll}
\overline{\mathbf{F}}_{N}=-\int_{\Gamma_{L}} \mathbf{N} p_{E} \cdot \mathbf{n} \mathrm{d} \Gamma_{L}+\mu_{E} \int_{\Gamma_{L}}\left(\nabla(\mathbf{N} \overline{\mathbf{v}})+\nabla(\mathbf{N} \overline{\mathbf{v}})^{T}\right)_{E} \cdot \mathbf{n} \mathrm{d} \Gamma_{L} & \text { on } \Gamma_{L} \\
\overline{\mathbf{F}}_{s t}=-\int_{\Gamma_{L}} \mathbf{N} \gamma \kappa \cdot \mathbf{n} \mathrm{d} \Gamma_{L} & \text { on } \Gamma_{L} \\
\overline{\mathbf{F}}_{s h}=\mu_{E} \int_{\Gamma_{L}}\left(\nabla(\mathbf{N} \overline{\mathbf{v}})+\nabla(\mathbf{N} \overline{\mathbf{v}})^{T}\right)_{E} \cdot \mathbf{m} \mathrm{d} \Gamma_{L} & (43)
\end{array}
$$

The integrands in the terms $\mathbf{F}_{N}$ and $\mathbf{F}_{s h}$ are computed in the interface elements of the Eulerian mesh and then are projected onto $\Gamma_{L}$, where the integrals are computed. The surface tension force is computed directly on the Lagrangian mesh.

\subsection{Solution algorithm}

To this end all the ingredients of the embedded Eulerian-Lagrangian multi-fluid formulation are defined. The overall solution of the coupled gas-liquid problem is presented next. Let us consider that at the time step $t_{n}$ the solution (velocity $\overline{\mathbf{v}}_{n}$ and pressure $\bar{p}_{n}$ ) is known in both the domains $\Omega_{E}$ and $\Omega_{L}$. To find the velocity and pressure fields at $t_{n+1}$ the following algorithm is implemented:

1. Solve the liquid problem using PFEM (Eq. (33)) and update the mesh position (Eq. 34).

Output: new position of the Lagrangian mesh, $\overline{\mathbf{v}}_{n+1} \bar{p}_{n+1}$ in $\Omega_{L}^{n+1}$

2. Identify the position of Lagrangian domain within the Eulerian one. Identify the interface elements, the "real" and the "fictitious" elements and nodes.

Output: $\Gamma_{I}$

3. In $\Omega_{E}$ : fix the velocity at the "real" nodes of the interface elements to the known gas velocity: $\overline{\mathbf{v}}_{r, n+1}=\overline{\mathbf{v}}_{n}$.

4. Solve the minimization problem (Eq. (38)) obtaining the velocity at the "fictitious" nodes of the interface elements $\overline{\mathbf{v}}_{f, n+1}$.

Fix the velocity at the fictitious nodes (interface Dirichlet b.c.).

5. "Switch off" the fictitious Eulerian elements $\left(\Omega_{E_{f}}\right)$.

6. Solve the gas problem (using real part of the Eulerian domain $\Omega_{E_{r}}$ ) (Eqs. (24), (25) and (26)) equipped with the interface Dirichlet boundary condition applied to the fictitious nodes.

Output: velocity and pressure $\overline{\mathbf{v}}_{n+1}$ and $\bar{p}_{n+1}$ in $\Omega_{E}^{n+1}$. 


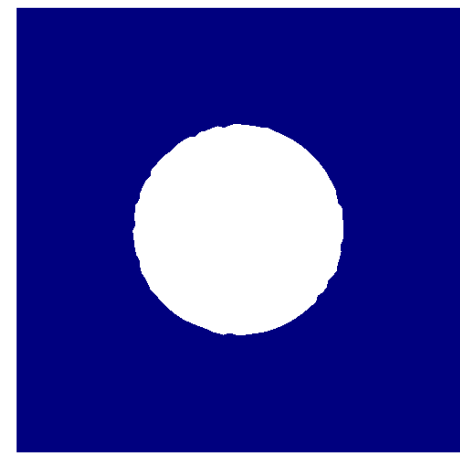

(a) Eulerian domain
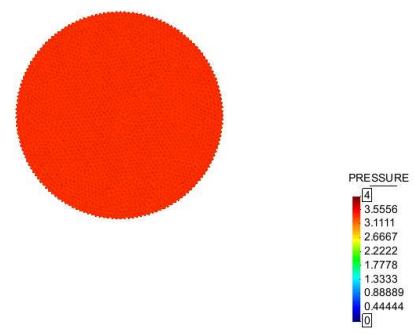

(b) Lagrangian domain

Figure 4: Pressure field at the final time step

7. Project the air stresses onto the liquid boundary $\Gamma_{L}$ surface and compute the corresponding force term $\overline{\mathbf{F}}_{\text {int }}$ (Eq. (40)) for the momentum equation of the liquid (29).

8. Go to next time step

An iterative version of the coupled scheme is obtained by repeating steps 1-7 until convergence in terms of the velocity of the Lagrangian boundary nodes of $\Gamma_{L}$ is achieved to the required precision $\epsilon: \frac{\|\delta \overline{\mathbf{v}}\|}{\left\|\overline{\mathbf{v}}_{n+1}\right\|}<\epsilon$.

\section{Examples}

The method proposed here was implemented within the Kratos Multi-Physics, a $\mathrm{C}++$ object oriented FE framework [29].

\subsection{Static droplet}

The first example models a circular liquid droplet (Lagrangian fluid $L$ ) immersed into gas (Eulerian fluid $E$ ) at rest. The surface tension force is the only acting force and gravity is neglected $(g=0)$. Both fluids have equal densities $\left(\rho_{E}=\right.$ $\left.\rho_{L}=1 \mathrm{~kg} \mathrm{~m}^{-3}\right)$, viscosities $\left(\mu_{E}=\mu_{L}=1 \mathrm{~kg} \mathrm{~m}^{-1} \mathrm{~s}^{-1}\right)$ and the corresponding surface tension coefficient is $\gamma=1 \mathrm{~N} \mathrm{~m}^{-1}$. The domain is defined by a square of $1 \times 1 \mathrm{~m}$ filled with fluid $E$, and a circular droplet of fluid $L$ with radius $R=0.25$ $\mathrm{m}$ at the center of the domain. The whole domain is meshed using triangular elements of size $h=1 / 25 \mathrm{~m}$. According to the Laplace-Young equation, the pressure jump across the interface between the fluids is $\Delta p=\gamma / R=4 \mathrm{~Pa}$ at the steady state. The result of the simulation of $1 \mathrm{~s}$ with a time step of $0.01 \mathrm{~s}$ is shown in Fig. 4.

Fig. 6 shows the pressure profile across the middle section of the domain. The analytical solution is compared with the one obtained using the purely Lagrangian multi-fluid formulation proposed in [12] and the present embedded method.

Results show that the pressure profile obtained with the presented method has better agreement with the exact solution than the one obtained with the 


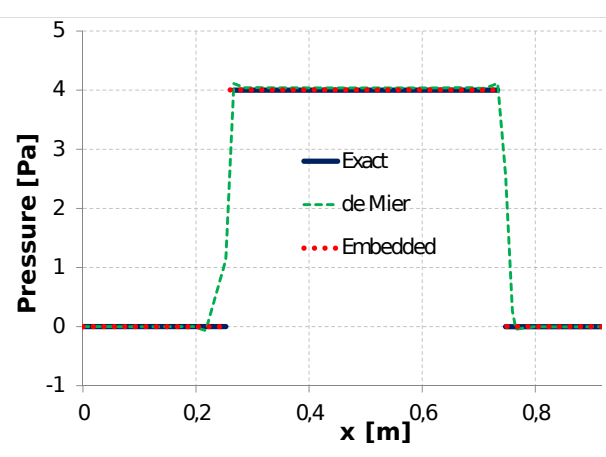

(a) Pressure profiles across the middle section

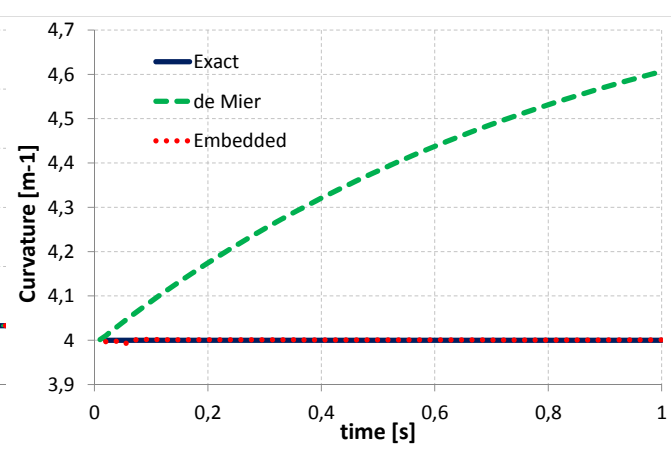

(b) Evolution of curvature over time

Figure 5: Comparison between the fully Lagrangian formulation ([12]), the embedded method and the analytical solution. $p_{E}^{\text {init }}=0 \mathrm{~Pa}$

fully Lagrangian formulation. The pressure discontinuity at the interface is represented exactly when using the present method.

De Mier [12] shows that if the pressure discontinuity is modeled by a continuous approximation with steep gradient, the steady state solution exhibits spurious velocities at the interface because the pressure gradient term dominates in the governing equation. This effect can be seen in 6 , where the pressure discontinuity is approximated by a continuous steep change across the interface elements. One can also observe slight under and over-shoots of pressure in the results corresponding the fully Lagrangian method [12]. The corresponding spurious velocities, in turn, lead to temporal evolution of curvature (which, according to the analytical solution must be constant). Thus the solution deviates from the exact one (constant). Fig. 5(b) shows the evolution of the curvature at an interface node with coordinates $(0.5,0.75)$ according to both models. The result obtained with the embedded formulation shows perfect agreement with the exact solution.

Solution schemes for the liquid phase The comparison of different solution schemes applied to the solution of the governing equations for the liquid phase is shown in Fig. 6. The example is solved for lower viscosity values ( $\mu=0.001 \mathrm{~kg} \mathrm{~m}^{-1} \mathrm{~s}^{-1}$ and $\mu=0.00001 \mathrm{~kg} \mathrm{~m}^{-1} \mathrm{~s}^{-1}$ were considered) in order to reduce the damping. The velocity at the boundary and the pressure at the first layer of nodes in the vicinity of the boundary are analyzed (the coordinates of the nodes are $(0.25,0.5)$ and $(0.29,0.5)$, respectively). The external pressure in the air is neglected $\left(p_{E}=0\right)$ and the only acting force is the surface tension $(\gamma \kappa=4 \mathrm{~Pa})$. The solutions obtained using the coupled velocity-pressure (monolithic) solver and the one of the fractional step solver are compared in Fig. 6(a). In the fractional step method, the pressure at the liquid surface is fixed. This "strong" way of imposing pressure violates the incompressibility condition, leading to the motion of the boundary. Thus, the curvature and therefore the surface tension force also deviate from the exact value. As a consequence, oscillations appear in the solution. For low viscosity values the oscillations become more pronounced. In the case of the coupled velocity-pressure (monolithic) 
solver no "artificial" pressure boundary condition is necessary. One can see an exact stable solution. Next, the surface surface tension $(\gamma=0)$ term is neglected, however external pressure $\left(p_{E}=4 P a\right)$ is applied. In this case both schemes show stable results (see Fig. 6(b)). Evolution of the velocity at a boundary node for different schemes is depicted in Fig. 6(c).
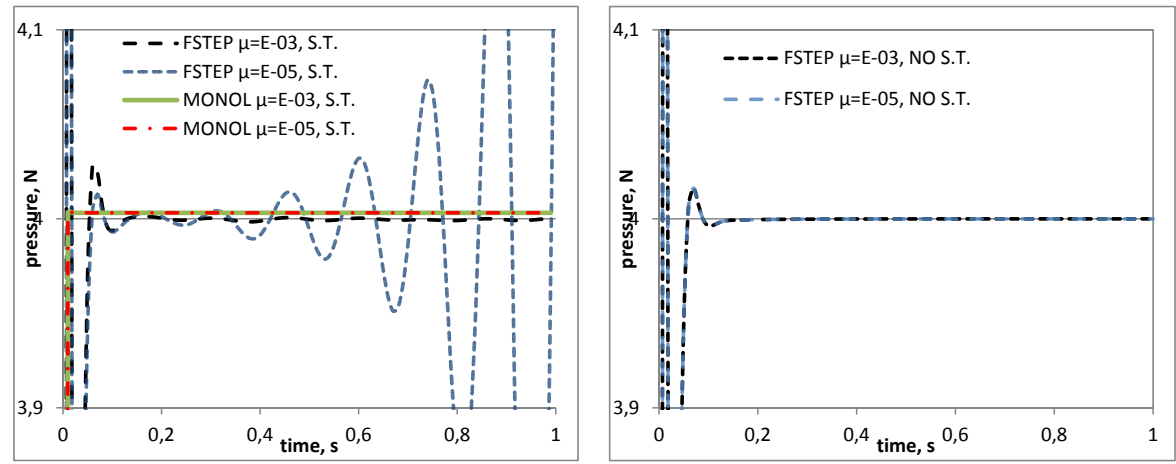

(a) Ext. pressure $p_{E}=0 \mathrm{~Pa}$, surf. tens. coef.(b) Ext. pressure $p_{E}=4 \mathrm{~Pa}$, surf. tens. coef. $\gamma=1$ $\gamma=0$

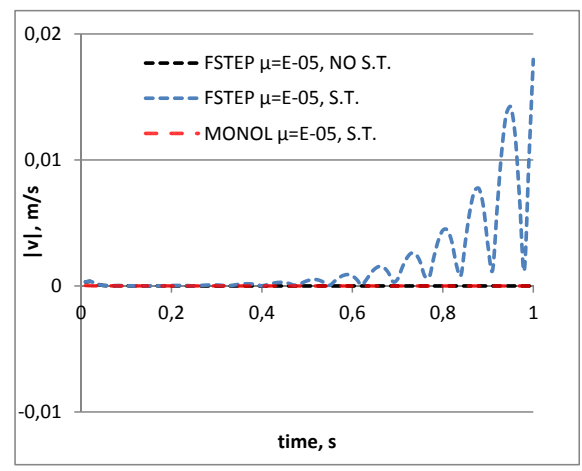

(c) Velocity at a boundary node

Figure 6: Comparison among the solutions obtained using the coupled (monolithic) and the fractional step scheme in the liquid phase.

Different initial conditions Next, the example is examined under different initial conditions. A non-zero initial pressure $(10 \mathrm{~Pa})$ is applied in fluid $E$. Zero initial pressure in fluid $L$ is maintained. According to Laplace-Young equation, the pressure jump must remain $\Delta p=p_{L}-p_{E}=\gamma / R=4 \mathrm{~Pa}$ at the steady state. To achieve this value the pressure in fluid $L$ must increase up to $14 \mathrm{~Pa}$. Fig. 


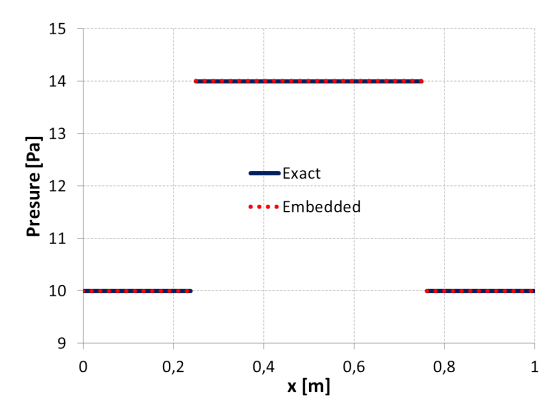

(a) Pressure profiles across the middle section

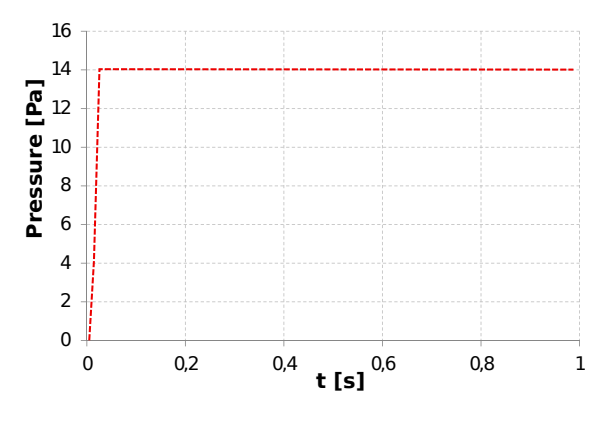

(b) Evolution of pressure at an inner node

Figure 7: Results for the non-zero initial pressure in the gas phase $\left(p_{E}^{\text {init }}=10\right.$ $\mathrm{Pa})$

7 (a) shows that the simulation results coincide with the analytical solution.

\subsection{Oscillation of free and levitating droplet}

This example examines the oscillatory behavior of a liquid droplet. When its initial shape is different from the equilibrium one (i.e., spherical if gravity was neglected) the droplet exhibits several oscillations prior to reaching the equilibrium state. Lamb (see p. 475 in [30]) performed an analytical study and found the expression for droplets' eigenfrequencies:

$$
f_{n}=\frac{1}{2 \pi} \sqrt{\frac{n(n-1)(n+2) \gamma}{\rho_{L} R^{3}}}
$$

where $n$ is the oscillation mode, $\rho_{L}$ is the liquid's density and $R$ is the droplet radius. Recently, the oscillatory droplet behavior and its interaction with an airflow was analyzed numerically by Bouwhuis et al. [31].

\subsubsection{Free droplet}

First, we examine the free oscillations of the droplet. The setup is similar to the one shown in the previous example: a water droplet is placed in the center of a square domain filled with air. However, the initial shape of the droplet is elliptical instead of spherical (Fig. 11(a)), with $a=1$ and $b=3 \mathrm{~mm}$, as proposed in [31]. The actual physical densities of water and air are used in this example. The gravity force is neglected. Two different values of water viscosity are used in order to check its effect on the resulting oscillations, and the time step is set to $10^{-5} \mathrm{~s}$.

According to Eq. (44) and considering the first non-zero oscillation mode $(\mathrm{n}=2)$, the frequency should be $\sim 43 \mathrm{~Hz}$. The values obtained using the present model $(f=46 \mathrm{~Hz})$ show a very good agreement with the analytical value. The predicted value shows good agreement with the numerical simulation results from [31] as well $(\sim 50 \mathrm{~Hz})$. It is important to note that both the viscosity and the time step used in the numerical simulations have to be sufficiently low in 


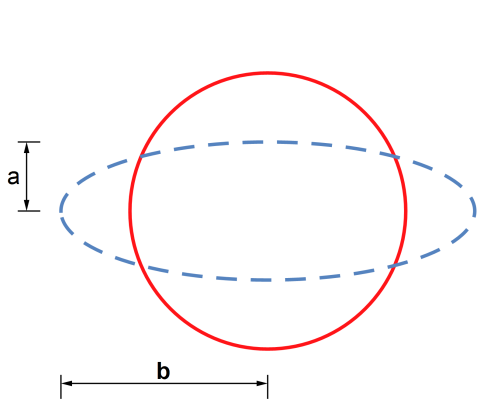

(a) Initial and final droplet shapes

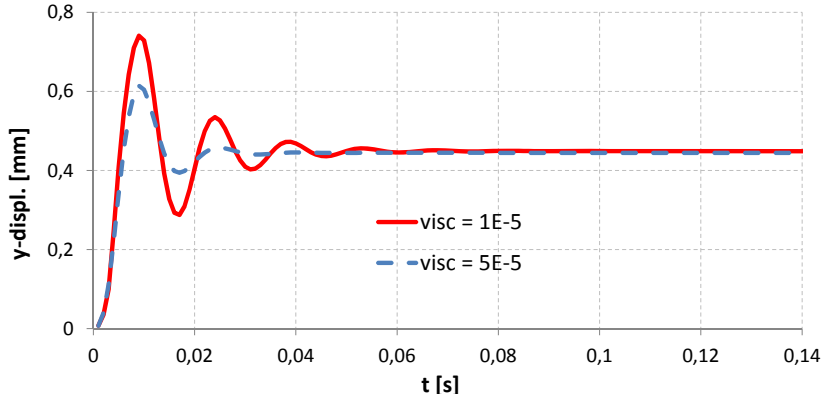

(b) Evolution of pressure over time

Figure 8: Shape and pressure evolution of an elliptic droplet with different viscosities

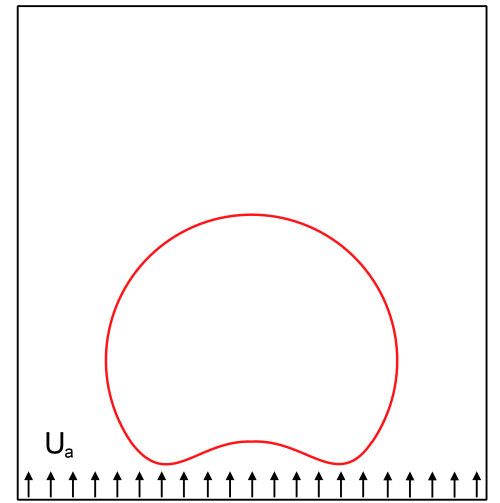

Figure 9: Drop levitated by an airflow entering the domain with uniform velocity $U_{a}$

order to observe oscillations in the air-water interface [31]. No oscillations may be detected for large viscosity values, confirming the observation of Bouwhuis.

\subsubsection{Droplet levitated by an airflow}

In this section the behavior of the droplet exposed to an airflow is studied. A liquid droplet is immersed in a rectangular channel of $10 \mathrm{~mm}$ width. A constant air flux is generated at the bottom of the channel. A schematic representation of the test case is shown in Fig. 9. The data is taken from [31].

For a given set of parameters the droplet levitates on top of the air cushion. At the beginning, the droplet tends to approach the channel bottom. As the droplet descends, the air velocity and pressure increase (the cushion is created) producing the desired effect of levitation.

The parameters describing the behavior of the droplet can be combined into 3 dimensionless numbers: the Bond number $B o$ (accounting for gravity against surface tension effects), the capillary number $C a$ (gas viscosity against surface tension) and the ratio between the dynamic viscosity of the gas and the liquid 
$\Lambda:$

$$
\begin{aligned}
& B o=\sqrt{\frac{\rho_{L} R^{2} g}{\gamma}} \\
& C a=\frac{\mu_{E} u_{E}}{\gamma} \\
& \Lambda=\frac{\mu_{L}}{\mu_{E}}
\end{aligned}
$$

where $R$ is the unperturbed droplet radius and $\mu_{L}$ and $\mu_{E}$ are the liquid and gas viscosities, respectively.

The simulation was performed for $B o=1, C a=2.5 \times 10^{-4}, \Lambda=11 \times 10^{3}$. The time step used in the simulations was $10^{-5} \mathrm{~s}$.

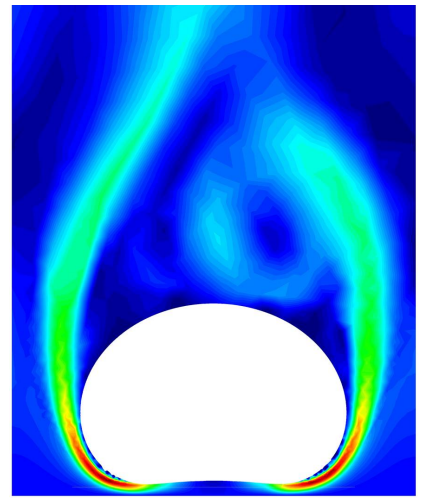

(a) $\mathrm{t}=65 \mathrm{~ms}$

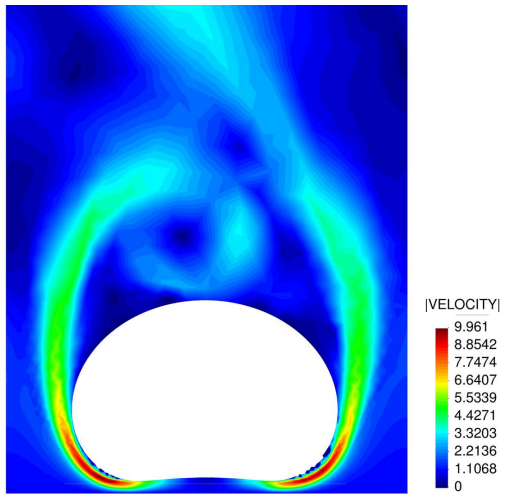

(b) $\mathrm{t}=75 \mathrm{~ms}$

Figure 10: Velocity field in the air domain

Fig. 10 shows the velocity pattern in the air domain. One can see the non-steady oscillatory nature of the airflow. In the present work the dynamic simulation was performed for both the gas and the liquid, while in [31] the inertial effects in the gas were neglected.

The mean droplet shape after the onset of the periodic oscillations and the corresponding air pressure across the "cushion" are shown in Fig. 11. Results are compared against the ones of [31].

The "chimney" effect is observed as expected (an air bubble develops below the drop and pierces the center of the droplet). The maximum pressure gradient coincides with the neck position. The obtained pressure distribution and the droplet shape closely resembles the results in [31].

\subsection{Two-fluid sloshing}

Two previous examples dealt with the problems where the effect of surface tension was dominating and the liquid domain was not undergoing severe deformations. Next, the embedded method is tested in application to the case where gravitational effects are dominating, the domain undergoes severe deformations and the surface tension effects can be neglected. 


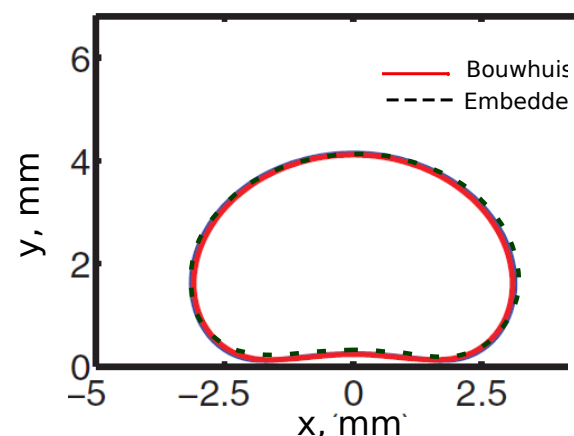

(a) Droplet shape

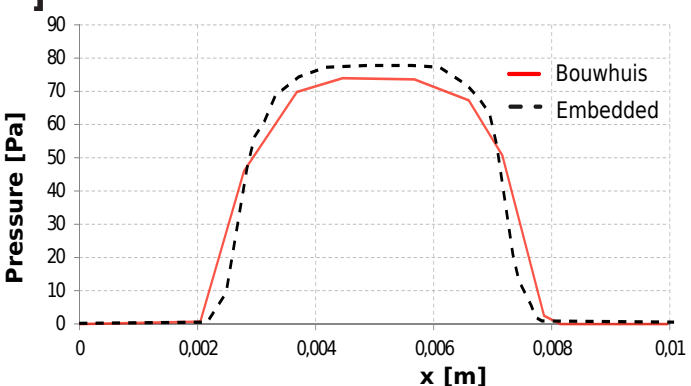

(b) Pressure profile in the air

Figure 11: Drop exposed to airflow.

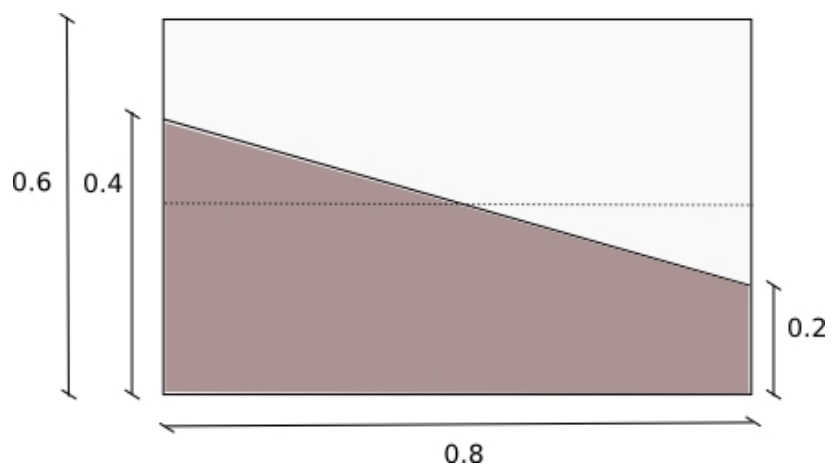

Figure 12: Two-fluid sloshing model (in meters)

This numerical test for two-fluid problems was proposed originally by Tezduyar et al. in [32], studied in detail by Cruchaga et al [5], [33] and recently by de Mier [12]. The computational domain consists of a closed container with the dimensions $0.8 \times 0.6 \mathrm{~m}$. The container is filled with two immiscible fluids, the lighter one being on top of the heavier one. The initial, inclined interface is linear with an average height of $0.3 \mathrm{~m}$. The fluid properties used in [32] are taken here as the reference parameters. The top fluid has density $\rho_{L}=1$ $\mathrm{kg} / \mathrm{m}^{3}$, the dynamic viscosity is constant $\mu_{E}=\mu_{L}=10^{-3} \mathrm{~Pa} \cdot \mathrm{s}$ in both fluids, and the gravity acceleration is set to $g=-0.294 \mathrm{~m} / \mathrm{s}^{2}$ in the vertical direction. The density value of the bottom fluid is varied. A no-slip condition is set at the horizontal walls, while at the vertical walls a slip condition is prescribed. Following the proposal of de Mier [12] the slip condition in the Lagrangian domain is modeled by considering viscosity $\mu_{L}=0$ at the elements encountered in contact with the wall.

Partitioning validation Prior to considering fluids with different densities, we test the capability of the proposed partitioned approach in representing the hydrostatic case $\left(\rho_{E}=\rho_{L}=1 \mathrm{~kg} / \mathrm{m}^{3}\right)$. The corresponding exact solution is zero velocity in the entire domain and the hydrostatic pressure distribution.

Before the coupling is applied, a non-zero velocity arises at the interface and 

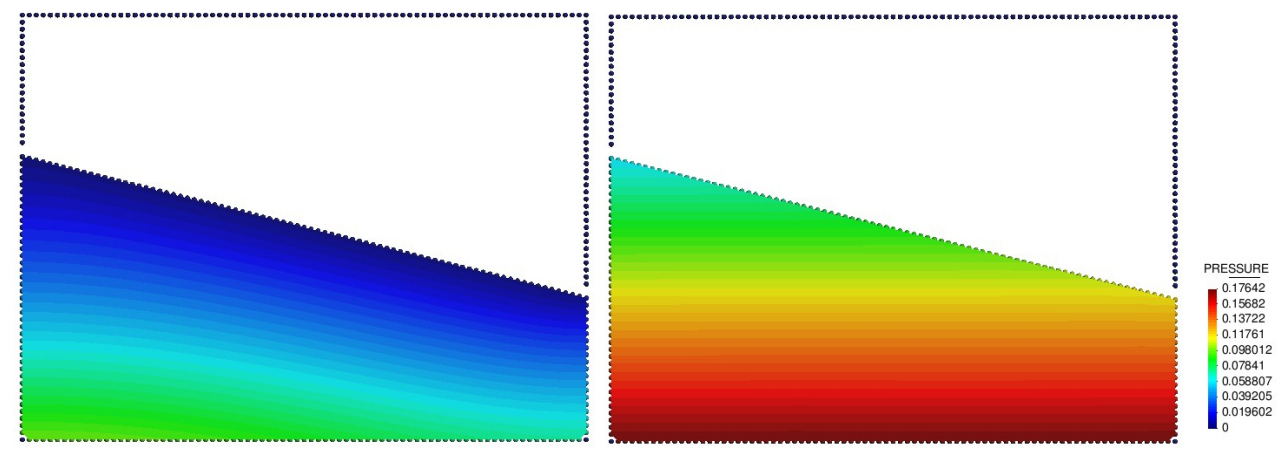

Figure 13: Pressure field in the Lagrangian domain for the density ratio 1:1. Prior to the application of the coupling (left) and after applying the coupling (right).

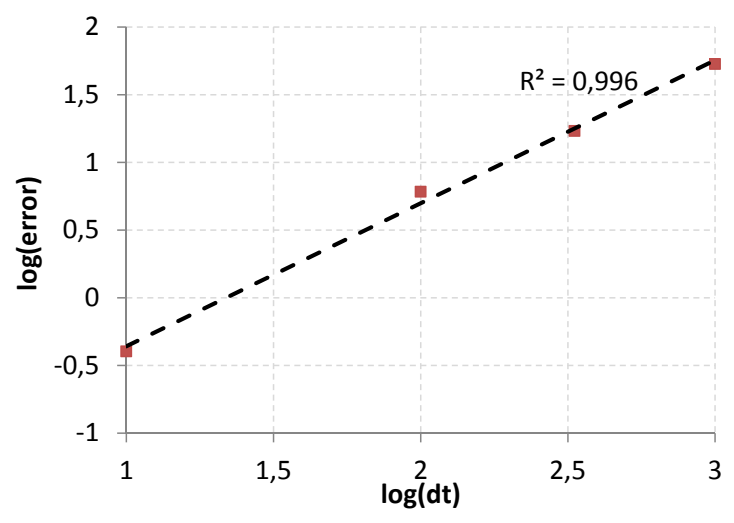

Figure 14: Error at $\mathrm{t}=0.5 \mathrm{~s}$ against time step

the pressure distribution is different from the hydrostatic one. However, the exact hydrostatic solution is recovered already in the first time step (see Fig. 13). The time step size used in the simulation was set to $0.01 \mathrm{~s}$.

Time accuracy The convergence rate is assessed by measuring the absolute error in the velocity as a function of time step size for the hydrostatic case. Four time step sizes were considered: $\mathrm{dt}=0.1 \mathrm{~s}, \mathrm{dt}=0.01 \mathrm{~s}, \mathrm{dt}=0.003 \mathrm{~s}, \mathrm{dt}=0.001 \mathrm{~s}$. Convergence of the time approximation is displayed in Fig. 14. The diminishing of the error with decreasing the time step is plotted. The error has been computed as the sum of the nodal errors at time $\mathrm{t}=0.5 \mathrm{~s}: \operatorname{Err}=\sum_{n}\left\|\mathbf{v}_{y}-\mathbf{v}_{y}^{\text {exact }}\right\|$ ( $\mathrm{n}$ is the number of nodes). Since the exact solution of the problem is the zero velocity in the entire domain, the error is simply the sum of nodal velocities.

One can see that the proposed method exhibits linear convergence rate.

Fluids with different densities Next, two fluids with different densities are considered. The densities of the bottom and the top fluids are $\rho_{L}=2 \mathrm{~kg} / \mathrm{m}^{3}$ and $\rho_{E}=1 \mathrm{~kg} / \mathrm{m}^{3}$, respectively. The unstructured uniform triangular FE meshes with approximately 20000 elements and the time step size of $d t=0.005 \mathrm{~s}$ were used if not mentioned otherwise. Fig. 15 displays the pressure fields at different 


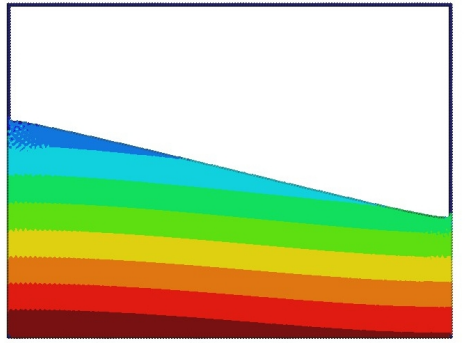

(a) Lagrangian part: $0.5 \mathrm{~s}$

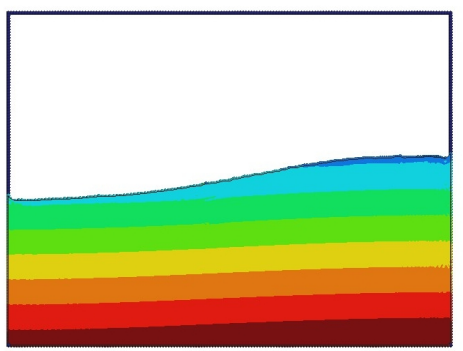

(c) Lagrangian part: $6 \mathrm{~s}$

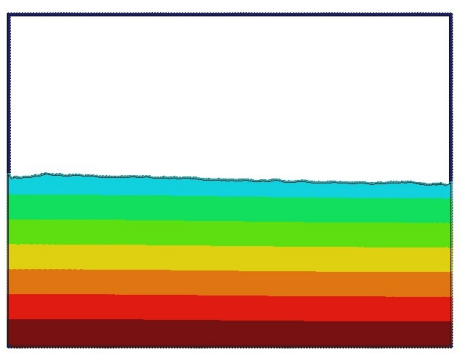

(e) Lagrangian part: $21 \mathrm{~s}$

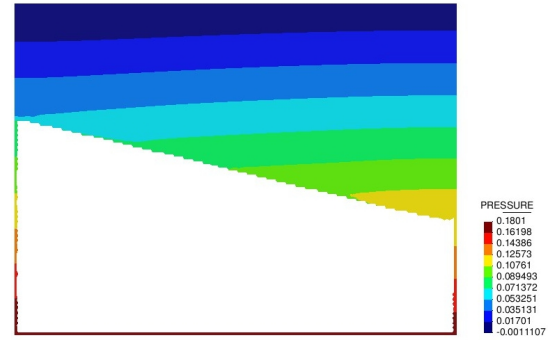

(b) Eulerian part: $0.5 \mathrm{~s}$

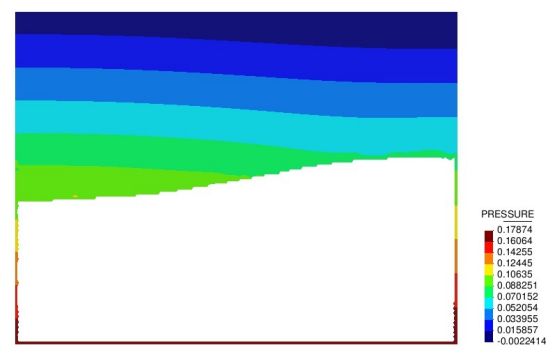

(d) Eulerian part: $6 \mathrm{~s}$

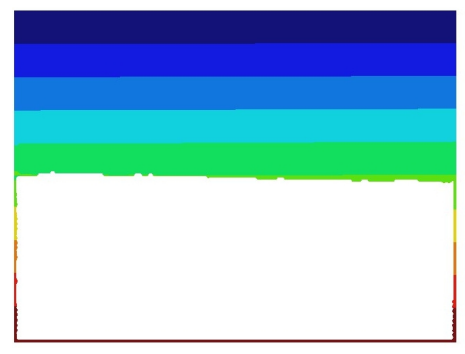

(f) Eulerian part: $21 \mathrm{~s}$

Figure 15: Pressure contours for two-fluid sloshing with density ratio 2:1

time instances for each domain. The Eulerian and the Lagrangian domains are juxtaposed.

Pressure distribution along the vertical cut made at the end of the simulation $(t=100 \mathrm{~s})$ is shown on Fig. 16. One can see the continuous pressure and the discontinuous pressure gradient at the interface due to density change. The pressure distribution is hydrostatic at the steady state.

Fig. 18(a) shows the evolution of the relative height of the interface $\left(h_{r e l}=\right.$ $\frac{h-0.3}{0.3}$. where $h_{r e l}$ and $h$ are the relative and absolute interface heights, respectively) at the left vertical wall, comparing the results obtained using the present formulations with the ones found in literature [5]. One can see a good agreement both in the amplitude evolution and the frequency. However, slight difference (around $10 \%$ ) in the period of oscillation is observed for this case. It is worth mentioning that Cruchaga et al. observed a slightly lower period of oscillation when their method was applied without the mass correction technique (see e.g. p. 8 in [5]). No mass correction was applied in the present work. 


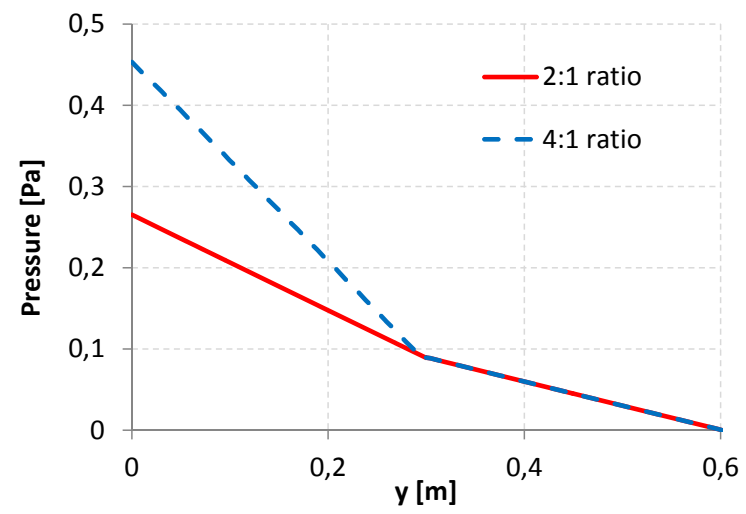

Figure 16: Pressure along the height $(\mathrm{x}=0.4 \mathrm{~m})$ at time $=0.5 \mathrm{~s}$

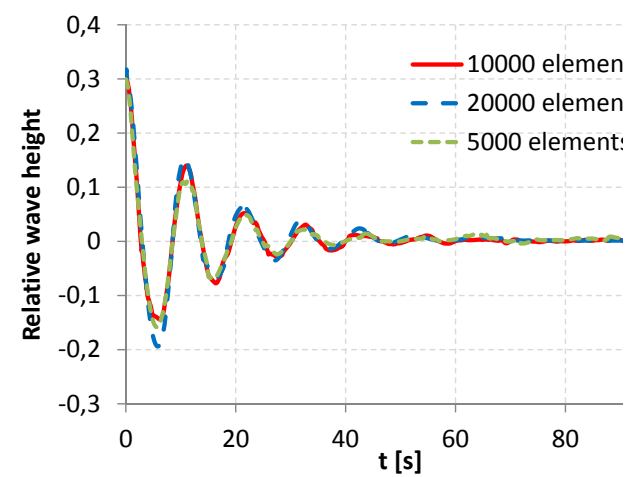

(a) Different mesh resolutions

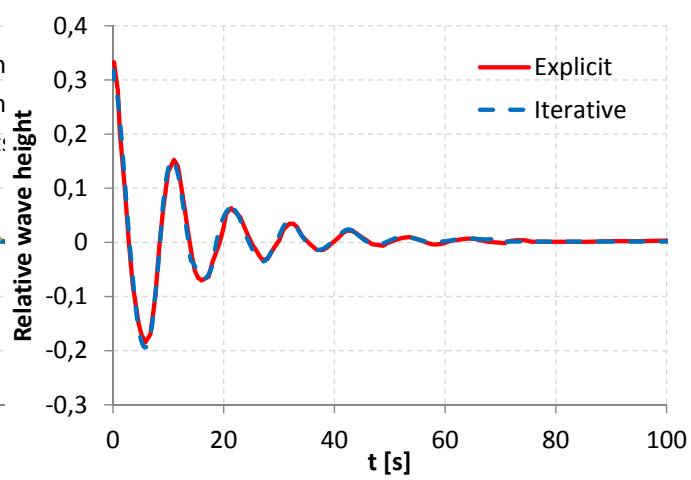

(b) Explicit vs. iterative

Figure 17: Liquid wave height at the left wall using different mesh sizes and time steps. Density ratio 2:1

Fig. 17(a) displays the comparison of the solutions obtained using three different meshes (5000, 10000 and 20000 elements). One can observe convergent solution. The solution obtained with the iterative version of the coupling (convergence tolerance was set to $\epsilon=10 E-06$ ) is compared with the staggered one.

comparison of the solutions obtained using time steps is shown in Fig. 17(b).

Next we examine the same example increasing the liquid density to $\rho_{L}=4$ $\mathrm{kg} / \mathrm{m}^{3}$ and maintaining the gas density as $\rho_{E}=1 \mathrm{~kg} / \mathrm{m}^{3}$. Fig. 18(b) shows the evolution of the relative height of the interface at the left vertical wall (comparison with the results reported in [5]). We observe increments in oscillation amplitude, frequency, and the time it takes to reach the equilibrium position. One can see a very good agreement with the results found in [5].

In application to this example our approach allowed to avoid a) interface distortions faced in the purely Lagrangian multi-fluid formulations (see e.g. p. 71 in [12]) b) necessity of using discontinuous pressure shape functions or enrichment for representing the pressure gradient discontinuity. It is worth mentioning 


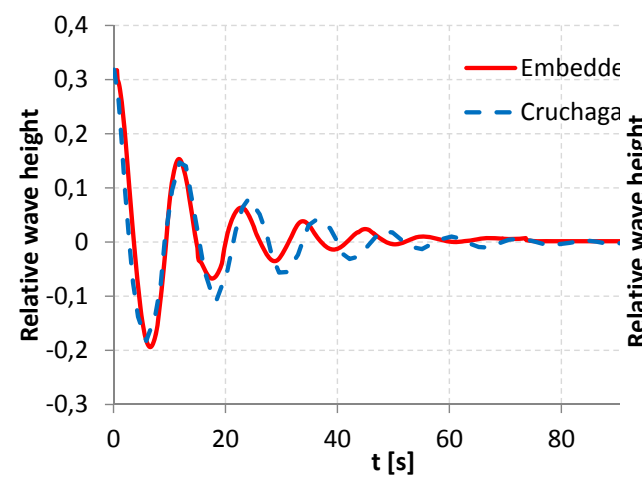

(a) Density ratio 2:1

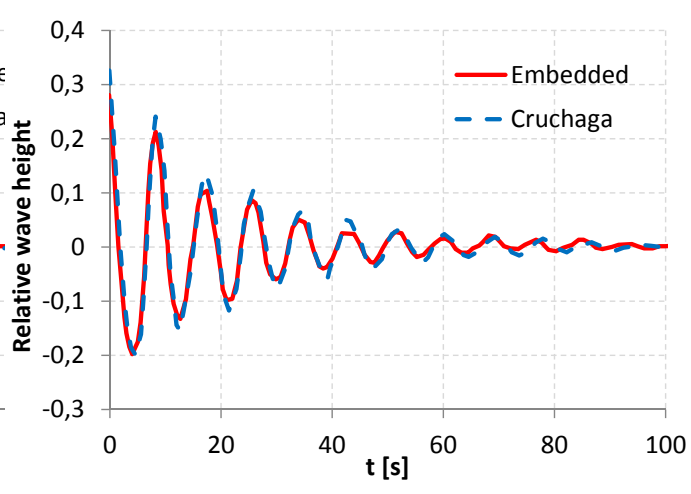

(b) Density ratio 4:1

Figure 18: Liquid wave height at the left wall compared to reference [5]

that the interface height at the steady state is coinciding with the theoretical one (0.3), giving an insight of a good overall mass conservation of the method. The volume change encountered at $t=100 \mathrm{~s}$ for the meshes containing 5000 and 20000 elements was of order of around $2 \%$ and $0.5 \%$, respectively.

\section{Summary and conclusions}

This paper presented an embedded formulation for gas-liquid systems based upon a combination of the Eulerian and the Lagrangian formulations. Gas was modeled by the Eulerian formulation, while the Lagrangian one was adopted for the liquid. The fluids were coupled using a Dirichlet-Neumann coupling. The interface Dirichlet boundary condition was satisfied in an integral sense minimizing the velocity difference of the two fluids across the interface. This condition was applied at the fictitious nodes of the interface elements of the Eulerian mesh. Normal and the shear stresses in the gas phase projected onto Lagrangian boundary mesh as well as the surface tension provided Neumann boundary condition for the liquid surface. Staggered approach for the solution of the coupled problem was proposed. An important advantage of the proposed embedded approach is that the interface position is exactly defined by the Lagrangian mesh. The interface maintains itself sharp without diffusion along time. The weak/strong pressure discontinuity along the interface due density change/surface tension is naturally accounted for by the method.

The formulation is particularly advantageous for the problems where the gas phase constitutes the major part of the overall computational domain. This allows to take the maximum advantage of the Lagrangian description for the liquid with changing boundaries while not increasing considerably the overall computational cost due to re-meshing or the use of the coupled velocity-pressure scheme.

Several test computations were carried out to evaluate the performance of the method. The formulation led to stable solutions for a wide range of the density ratios of the fluids involved. It was found that for the staggered version of the 
formulation is first order accurate in time. It was observed that including the viscous stress in the coupling is essential in the problems dealing with droplets exposed to the air flux.

The main advantages of the formulation are

- Modular approach

- Natural representation of the pressure discontinuity across the interface

- Application of Dirichlet boundary condition in an integral way using the fictitious nodes of the interface elements

- Additional techniques (typical for fully Eulerian formulations) for interface tracking are not necessary

- Absence of problems with interface preservation (typical for the purely Lagrangian multi-fluid formulations)

- Good mass conservation

Keeping in mind all the advantages of the formulation, it is important to note that it also has some limitations. For optimal functionality of the method the mesh sizes of the involved domains should be similar. This precludes the use of highly heterogeneous meshes. Time step size is generally restricted due to the danger of the element inversion faced in the classical PFEM method used for the liquid in the present work. Modeling the liquid domain using a novel Lagrangian formulation based on explicit stream-line temporal integration [34] is a promising alternative that must be studied in the future. While keeping the overall "architecture" of the approach proposed here, this alternative Lagrangian formulation may lead to considerable advantages in computational efficiency.

Coupled velocity-pressure scheme used in the present work for the liquid phase was chosen in order to avoid the artificial pressure boundary condition necessary in the standard fractional step schemes. This was found particularly important for the problems where surface tension effects cannot be neglected. In such cases standard fractional step schemes led to spurious oscillations of the interface. Alternatives to the coupled scheme such as e.g. incremental pressurecorrection scheme in rotational form that provides the consistent boundary condition for the pressure [21], [35] must be investigated. Possibility of using this approach in both domains and its impact on the computational efficiency will be investigated in the future.

\section{Acknowledgements}

This work was supported under the auspices of the uLites project of the European Commission (FP7-SME-2012 GA-314891), the COMETAD project of the National RTD Plan (ref. MAT2014-60435-C2-1-R) and the FPI Research Grant BES-2011-047702 subject to the Spanish Project BIA2010-15880, from the Ministerio de Economía y Competitividad. 


\section{References}

[1] S J Osher and R P Fedkiw. Level Set Methods and Dynamic Implicit Surfaces. Springer, 2006.

[2] R Rossi, A Larese, P Dadvand, and E Oñate. An efficient edge-based level set finite element method for free surface flow problems. International Journal for Numerical Methods in Fluids, 71(6):687-716, 2013.

[3] H W Cyril and B D Nichols. Volume of fluid (VOF) method for the dynamics of free boundaries. Journal of computational physics, 39(1):201-225, 1981.

[4] M Cruchaga, E Oñate, and S Idelsohn. On the pseudomaterial approach for the analysis of transient forming processes. Communications in numerical methods in engineering, 11(2):137-148, 1995.

[5] M Cruchaga, D Celentano, and T Tezduyar. A moving Lagrangian interface technique for flow computations over fixed meshes. Computer Methods in Applied Mechanics and Engineering, 191(6):525-543, 2001.

[6] P T Barton, B Obadia, and D Drikakis. A conservative level-set method for compressible solid/fluid problems on fixed grids. Journal of Computational Physics, 230:7867-7890, 2011.

[7] P D M Spelt. A level-set approach for simulations of flows with multiple moving contact lines with hysteresis. Journal of Computational Physics, 207(2):389-404, 2005.

[8] Y L Zhang, Q P Zou, and D Greaves. Numerical simulation of free-surface flow using the level-set method with global mass correction. International Journal of Numerical Methods in Fluids, 63(6):366-396, 2010.

[9] R F Ausas, E A Dari, and G C Buscaglia. A geometric mass-preservating redistancing scheme for the level set function. International Journal of Numerical Methods in Fluids, 65(8):989-1010, 2011.

[10] A H Coppola-Owen and R Codina. Improving Eulerian two-phase flow finite element approximation with discontinuous gradient pressure shape functions. International Journal for Numerical Methods in Fluids, 49(12):12871304, 2005.

[11] S Idelsohn, M de Mier-Torrecilla, and E Oñate. Multi-fluid flows with the particle finite element method. Computer Methods in Applied Mechanics and Engineering, 198(33):2750-2767, 2009.

[12] M Mier-Torrecilla. Numerical simulation of multi-fluid flows with the Particle Finite Element Method. PhD thesis, Universitat Politècnica de Catalunya, 2010.

[13] A Huerta and W K Liu. Viscous flow with large free surface motion. Computer Methods in Applied Mechanics and Engineering, 69(3):277-324, 1988. 
[14] A Gerstenberger and W Wall. An eXtended Finite Element/Lagrange multiplier based approach for fluid-structure interaction. Computer Methods in Applied Mechanics and Engineering, 197:1699-1714, 2008.

[15] E Oñate, S Idelsohn, F del Pin, and R Aubry. The Particle Finite Element Method: an overview. International Journal of Computational Methods, 1:267-307, 2004.

[16] J Donea and A Huerta. Finite element method for flow problems. J. Wiley, 2003.

[17] R Löhner. Applied CFD Techniques, 2nd edition. J. Wiley and Sons, 2008.

[18] Codina R. A stabilized finite element method for generalized stationary incompressible flows. Computer Methods in Applied Mechanics and Engineering, 190(20-21):2681 - 2706, 2001.

[19] A J Chorin. A numerical method for solving incompressible viscous problems. Journal of Computational Physics, 2:12-26, 1967.

[20] R Temam. Sur lapproximation de la solution des equations de Navier-Stokes par la methode des pase fractionaires. Archives for Rational Mechanics and Analysis, 32:135-153, 1969.

[21] Guermond J.L., Minev P., and Shen J. An overview of projection methods for incompressible flows. Computer Methods in Applied Mechanics and Engineering, 195:6011-6045, 2006.

[22] R Codina. Pressure stability in fractional step finite element method for incompressible flows. Journal of Computational Physics, 170:112-140, 2001.

[23] S Idelsohn, E Oñate, and F del Pin. The Particle Finite Element Method: a powerful tool to solve incompressible flows with free-surfaces and breaking waves. International Journal of Numerical Methods in Engineering, 61:964989, 2004.

[24] B Delaunay. Sur la sphre vide. Izvestia Akademii Nauk SSSR, Otdelenie Matematicheskikh i Estestvennykh Nauk, 7:793-800, 1934.

[25] P Ryzhakov, R Rossi, S Idelsohn, and E Oñate. A monolithic Lagrangian approach for fluid-structure interaction problems. Journal of Computational Mechanics, 46/6:883-399, 2010.

[26] R Codina, G Houzeaux, H Coppola-Owen, and J Baiges. The fixed-mesh ALE approach for the numerical approximation of flows in moving domains. Journal of Computational Physics archive, 228:1591-1611, 2009.

[27] P Ryzhakov, E Oñate, R Rossi, and S Idelsohn. Lagrangian FE methods for coupled problems in fluid mechanics. CIMNE, 2010.

[28] R Codina, G Houzeaux, H Coppola-Owen, and J Baiges. The fixed-mesh ALE approach for the numerical approximation of flows in moving domains. Journal of Computational Physics, 228(5):1591 - 1611, 2009. 
[29] P Dadvand, R Rossi, and E Oñate. An object-oriented environment for developing finite element codes for multi-disciplinary applications. Archieves of Computational Methods in Engineering, 17/3:253-297, 2010.

[30] H. Lamb. Hydrodynamics. University Press, 1895.

[31] W Bouwhuis, K G Winkels, I R Peters, P Brunet, D van der Meer, and $\mathrm{J} \mathrm{H}$ Snoeijer. Oscillating and star-shaped drops levitated by an airflow. Physical Review E, 88:023017, 2013.

[32] T Tezduyar, M Behr, S Mittal, and J Liou. A new strategy for finite element computations involving moving boundaries and interfacesthe deformingspatial-domain/space-time procedure: II. computation of free-surface flows, two-liquid flows, and flows with drifting cylinders. Computer methods in applied mechanics and engineering, 94(3):353-371, 1992.

[33] M Cruchaga and E Oñate. A generalized streamline finite element approach for the analysis of incompressible flow problems including moving surfaces. Computer Methods in Applied Mechanics and Engineering, 173(1):241-255, 1999.

[34] S Idelsohn, N Nigro, J M Gimenez, R Rossi, and J Marti. A fast and accurate method to solve the incompressible Navier-Stokes equations. Engineering Computations, 30(2):197-222, 2013.

[35] Guermond J.L. and Shen J. On the error estimates for the rotational pressure-correction projection methods. Mathematics of Computation, 73(248):1719-1737, 2004. 